\title{
Numerical modelling of waves in double-porosity Biot medium ${ }^{\text {is }}$
}

\author{
Eduard Rohan $^{\mathrm{a}, *}$, Vu-Hieu Nguyen ${ }^{\mathrm{b}}$, Salah Naili ${ }^{\mathrm{b}}$ \\ ${ }^{a}$ European Centre of Excellence, NTIS - New Technologies for Information Society Faculty of Applied Sciences, University of West Bohemia, Technická 2967, 30614 Pilsen, \\ Czech Republic \\ ${ }^{\mathrm{b}}$ Université Paris-Est, Laboratoire Modélisation et Simulation Multi Echelle, MSME UMR 8208 CNRS, 61 avenue du Général de Gaulle, 94010 Créteil cedex, France
}

\section{A R T I C L E I N F O}

Article history:

Accepted 14 September 2017

Available online 12 October 2017

\section{Keywords:}

Poroelasticity

Biot model

Homogenization

Double-porosity

Wave propagation

Numerical simulation

\begin{abstract}
A B S T R A C T
We consider acoustic waves in fluid-saturated periodic media with large contrasts in the permeability and other poroelastic coefficients, whereby some of the mesoscopic material parameters depend on the scale parameter. The effective behaviour is described by a model obtained using the homogenization of the heterogeneous Biot continuum relevant to the mesoscopic level at which the dual porosity is featured by low permeability, whereas the primary porosity is very compliant. All material coefficients of the homogenized model depend on the frequency of incident waves. The macroscopic fields can be employed to reconstruct solutions at the mesoscopic level relevant to the heterogeneities. To validate the model, we consider heterogeneous strips with a finite scale lattice defining the heterogeneity. For such structures, the recovered mesoscopic response computed using the numerical homogenization method is compared with direct numerical simulations for various contrasts. For media featured by large contrast the double porosity model provides much better estimates of the wavelengths and other dispersion properties than the standard single porosity homogenized model. Moreover, the performed tests show computational efficiency of the two-scale simulations using the homogenized model, while the direct simulations of a heterogeneous medium become unfeasible because of requirements on the finite element discretization.
\end{abstract}

(c) 2017 Elsevier Ltd. All rights reserved.

\section{Introduction}

Numerical simulations of wave propagation in heterogeneous materials belong to quite challenging issues in mathematical modelling and computational mechanics. On one hand, there are important applications in civil engineering, biomechanics, material and environmental sciences, on the other, the numerical methods and computational tools which are well developed to treat static, or quasistatic problems become much less efficient and may provide inaccurate results when employed straightforwardly to simulate wave responses of heterogeneous media. A possible remedy is to use averaging and upscaling techniques which, in principle, lead to models parameterized by effective material constants describing macroscopic behaviour of the medium. In the context of fluid saturated poroelastic media (FSPM), although the topic has been studied over the past decades and several modelling approaches and particular models have been proposed, the dispersion phenomenon has not been fully understood. The FSPM are constituted

\footnotetext{
Paper submitted to Computers \& Structures (SI devoted to the Conf. SEMC 2016).

* Corresponding author.

E-mail address: rohan@kme.zcu.cz (E. Rohan).
}

by a solid (elastic) skeleton in which the fluid-saturated pores are distributed as a connected pore network. The mathematical model governing the wave propagation in FSPM was first proposed by Biot $[5,6]$. The model equations were extended to include the most general case of anisotropy for a porous elastic solid with particular emphasis on viscoelasticity properties and relaxation effects [7]. Then the equations of the Biot model have been confirmed by the homogenization theory which is based on the asymptotic analysis of the fluid-structure interaction problem at the pore level [9], see e.g. the book of Auriault et al. [4] emphasizing the mechanical point of view, or mathematical references Hornung [14], Clopeau et al. [10], Ferrín and Mikelić [12].

Many natural, as well as artificial porous materials exhibit the presence of heterogeneity at scales much larger than microstructure scales, but much smaller than the wavelengths. For modelling of the wave propagation in such heterogeneous media, continuum porous model with spatially varying coefficients characterizing the mesoscopic structure may be used. We remark that homogenization of fractured porous media, as treated e.g. in Auriault and Boutin [2], is concerned with a different kind of mesoscopic structures leading to different models.

Here we report on modelling of the homogenized periodic mixture of two different porous media situated in two disjoint 
subdomains $\Omega_{c}$ and $\Omega_{m}$ which are distinguishable at the mesoscopic scale due to large contrasts between material coefficients characterizing their mechanical properties. To capture the behaviour of such a FSPM with large contrasts in the permeability and the poroelastic coefficients at the mesoscopic scale, the "single porosity" type models [15] may not provide a convenient approximation. In the present situation, the medium is described by the Biot model which is characterized by large contrasts in material properties of its mesoscopic constituents periodically distributed in the two porosities, called the matrix (the "dual" porosity) and the channels (the "primary" porosity). We adhere to the "matrix-channels" decomposition employed in our preceding publication related to a similar kind of the material with large contrasts, [19], cf. [20]. By virtue of the homogenization, this contrast is related to the scale parameter which is subject of the asymptotic analysis, cf. [1]. To explain the motivation for such a scaling, in the matrix, the poroelastic material is much less permeable than the one in the channels; this assumption leads to the scaledependent permeability in the matrix. While the channels are characterized by a large permeability, the solid skeleton is much more compliant than the one of the matrix, which leads to a reciprocal scaling of the elasticity in the channels. We analyze numerically properties of such a material using the homogenized model developed recently in [20] which is the extension of our previous works $[18,16]$ related to the rigid double porosity media. In [16], the model of rigid double porous materials was validated using direct numerical finite element simulations as the reference model. In particular, the two-scale solutions obtained using the homogenized model were compared with those computed using the mesoscopic model on the periodic heterogeneous structure represented by a finite element (FE) mesh generated by copies of the representative cell.

The present paper aims to validate the homogenized model of the "large contrast" Biot medium derived using the homogenization, see [20]. We explain how the macroscopic fields can be employed to reconstruct two-scale solutions at the mesocopic level relevant to the heterogeneities. For this, the characteristic responses are employed. To validate the model, we consider a heterogeneous layer of finite thickness with a finite scale lattice defining the heterogeneity. Assuming an incident plane wave, the computational domain is restricted to a column representing the layer. For such a structure, the recovered mesoscopic response computed using the numerical homogenization method is compared with direct numerical simulations in the heterogeneous medium. Moreover, in the proposed test, we consider different compositions of the periodic porous structure characterized by the contrast between mechanical properties of two different microporous materials. Besides the double porosity model, also the above mentioned single porosity model is used to solve the wave response of the layer, so that responses of the two homogenized models can be compared with the direct simulations for varying contrast property of the double porosity medium.

\subsection{Plan of the paper}

In Section 2 we introduce problem formulations for the wave propagation problem in the Biot medium relevant to the mesoscopic scale of the heterogeneity. The homogenized model of the double porosity medium is reported in Section 3, where formulations of the local problems for the so-called characteristic responses of the representative volume element (RVE) and formulae for the effective medium parameters are given. The two-scale reconstruction of the macroscopic solutions of the homogenized problem is described in Section 4. In Section 5, the validation tests are reported; for a given frequency of an incident wave, we compute responses of the heterogeneous media for several combinations of different microporous material, whereby solutions of the double and single porosity models are compared with solutions of the reference model. The results are discussed in the context of the material contrast. In Appendix A, the single porosity model is briefly presented to make the paper self-consistent.

\subsection{Notation}

In the paper, the mathematical models are formulated in a Cartesian framework of reference $\mathcal{R}\left(O ; \boldsymbol{e}_{1}, \boldsymbol{e}_{2}, \boldsymbol{e}_{3}\right)$ where $O$ is the origin of the space and $\left(\boldsymbol{e}_{1}, \boldsymbol{e}_{2}, \boldsymbol{e}_{3}\right)$ is a orthonormal basis for this space. The coordinates of a point $M$ are specified by $x=\left(x_{1}, x_{2}, x_{3}\right)$ in $\mathcal{R}$. The usual boldface notation for vectors $\boldsymbol{a}=\left(a_{i}\right)=\left(a_{1}, a_{2}, a_{3}\right)$ and second-order tensors $\boldsymbol{b}=\left(b_{i j}\right)$ is used. The gradient and divergence operators are respectively denoted by $\nabla$ and $\nabla \cdot$. When these operators have a subscript which is space variable, it is for indicating that the operator acts relatively at this space variable, for instance $\nabla_{x}=\left(\partial_{i}^{x}\right)$. The symbol dot ' ' denotes the scalar product between two vectors and the symbol colon ':' stands for scalar (inner) product of two second-order tensors. Standard notations for functional spaces are adhered. Throughout the paper, $x$ denotes the global ("macroscopic") coordinate, while the "local" coordinate $y$ describes the position within the representative unit cell $Y \subset \mathbb{R}^{3}$ where $\mathbb{R}$ is the set of real numbers.

\section{Waves propagating fluid saturated porous media}

We consider the monochromatic wave propagation in a fluidsaturated porous medium (FSPM) which is described at a scale where individual fluid-filled pores are not distinguishable so that at any point of the bulk material both the solid and fluid phases are present according to the volume fraction.

\subsection{The Biot model}

The model of wave propagation in the FSPM proposed by Biot $[5,6]$ provides the basis for our mathematical treatment of the double porosity media. For a given angular frequency $\omega$, the amplitudes of displacement $\boldsymbol{u}$, fluid pressure $p$ and seepage velocity $\boldsymbol{w}$ satisfy the following system of equations (where $\mathrm{i}^{2}=-1$ ),

$$
\begin{aligned}
& -\nabla \cdot[\mathbb{D} \boldsymbol{e}(\boldsymbol{u})-\boldsymbol{\alpha} p]-\omega^{2} \bar{\rho} \boldsymbol{u}+\mathrm{i} \omega \rho^{f} \boldsymbol{w}=\boldsymbol{0}, \\
& -\omega^{2} \rho^{f} \boldsymbol{u}+\eta[\boldsymbol{k}(\omega)]^{-1} \boldsymbol{w}+\nabla p=\boldsymbol{0}, \\
& \mathrm{i} \omega \boldsymbol{\alpha}: \boldsymbol{e}(\boldsymbol{u})+\nabla \cdot \boldsymbol{w}+\frac{\mathrm{i} \omega}{\mu} p=0,
\end{aligned}
$$

consisting of the momentum Eq. $(2.1)_{1}$, the generalized Darcy law $(2.1)_{2}$, and the fluid volume conservation $(2.1)_{3}$. We introduce the notation: $\boldsymbol{e}(\boldsymbol{u})$ is the small strain tensor, $\mathbb{D}$ is the skeleton elasticity tensor, tensor $\alpha$ is the Biot's coefficient of the effective stress, $1 / \mu$ is the Biot's compressibility coefficient, $\bar{\rho}=\rho^{s}\left(1-\phi_{0}\right)+\phi_{0} \rho^{f}$ is the local mean density given by the reference volume fraction $\phi_{0}$ of the fluid contents in the microporosity, the fluid density $\rho^{f}$, and by the solid phase density $\rho^{s}$.

In $[17,3,16]$, it has been shown that within the low frequency bands, the viscodynamic operator $\eta[\boldsymbol{k}(\omega)]^{-1}$, where $\eta$ is the fluid dynamic viscosity, can be expressed using the intrinsic static permeability $\boldsymbol{K}$ and density $\boldsymbol{\rho}$ which takes into account the tortuosity effects related to the kinetic energy, so that,

$\frac{1}{\eta} \boldsymbol{k}(\omega)=\left(\mathrm{i} \omega \boldsymbol{\rho}+[\boldsymbol{K}]^{-1}\right)^{-1}=: \boldsymbol{H}(\omega)$,

where $\boldsymbol{H}(\omega)$ is called the dynamic permeability. Both $\boldsymbol{\rho}$ and $\boldsymbol{K}$ are real-valued second-order tensors independent of the frequency. In isotropic media, $\boldsymbol{\rho}=\boldsymbol{I} \rho^{f} a_{\infty} / \phi_{0}$, where $a_{\infty}$ is the tortuosity parameter. For higher $\omega$, the decomposed form (2.2) provides merely an approximation, however, in this study we merit rather situations corresponding to the low frequency waves. Although, due to the 
large contrast in the elasticity coefficients, at the mesoscopic level of the heterogeneity, higher mode oscillations appear in the softer part, these modes are still associated with the monochromatic low frequency excitation. In this context, the high frequency treatment of the heterogeneous media proposed in [11] should not be confused with the approach reported here.

\subsection{Problem formulation for heterogeneous media}

We consider the system of Eq. (2.1) in domain $\Omega$ and the boundary conditions (BCs) on $\partial \boldsymbol{\Omega}$. We consider the boundary decomposition $\partial \boldsymbol{\Omega}=\partial_{p} \Omega \cup \partial_{w} \Omega$, where $\partial_{p} \Omega \cap \partial_{w} \Omega=\varnothing$ and another decomposition $\partial \boldsymbol{\Omega}=\partial_{u} \boldsymbol{\Omega} \cup \partial_{\sigma} \Omega$, where $\partial_{u} \boldsymbol{\Omega} \cap \partial_{\sigma} \boldsymbol{\Omega}=\varnothing$. The following BCs can be prescribed, in general (for a fixed real frequency $\omega \in \mathbb{R}$ ),

$\boldsymbol{u}=\overline{\boldsymbol{u}} \quad$ on $\quad \partial_{u} \Omega, \quad \boldsymbol{\sigma} \cdot \boldsymbol{n}=\mathbf{g} \quad$ on $\partial_{\sigma} \Omega$,

$p=\bar{p} \quad$ on $\quad \partial_{p} \Omega, \quad \boldsymbol{w} \cdot \boldsymbol{n}=0 \quad$ on $\quad \partial_{\boldsymbol{w}} \Omega$,

However, in what follows, for simplicity, we shall consider a special situation of undrained poroelastic material loaded by traction forces $\mathbf{g}$ and subject to a kinematic loading by $\overline{\boldsymbol{u}}$ on the nonempty part $\partial_{u} \Omega \neq \varnothing$, whereas $\partial_{p} \Omega=\varnothing$.

The boundary value problem can be reformulated in a weak sense using Sobolev spaces on $\Omega$. For this, we shall need the following spaces,

$\mathbf{H}_{0 /}^{1}(\Omega):=\left\{\boldsymbol{v} \in \mathbf{H}^{1}(\Omega) \mid \boldsymbol{u}=\mathbf{0} \quad\right.$ on $\left.\quad \partial_{u} \Omega\right\}$,

$\mathbf{H}_{0}(\operatorname{div}, \Omega):=\left\{\boldsymbol{v} \in \mathbf{L}^{2}(\Omega) \mid \nabla \cdot \boldsymbol{w} \in L^{2}(\Omega), \boldsymbol{w} \cdot \boldsymbol{n}=0 \quad\right.$ on $\left.\quad \partial \Omega\right\}$,

where the boldface notation is used for spaces of vectorial functions: $\mathbf{H}^{1}(\Omega)$ is associated with the Sobolev space $W^{1,2}(\Omega)$ and $\mathbf{L}^{2}(\boldsymbol{\Omega})$ is associated with the Lebesgue space $L^{2}(\Omega)$, see e.g. [8]. By $\mathbf{H}_{\overline{\boldsymbol{u}}}^{1}(\Omega) \subset \mathbf{H}^{1}(\Omega)$ we denote the set formed by functions satisfying the boundary condition for $\boldsymbol{u}$, see $(2.3)_{1}$, thus $\mathbf{H}_{\overline{\boldsymbol{u}}}^{1}(\boldsymbol{\Omega})=\mathbf{H}_{0 /(}^{1}(\boldsymbol{\Omega})+\overline{\boldsymbol{u}}$ (in the sense of an extension of $\overline{\boldsymbol{u}}$ to $\Omega$ ).

\section{Weak formulation}

The system (2.1) and (2.3) gives rise to the variational formulation of the wave propagation problem: Find $(\boldsymbol{u}, \boldsymbol{w}, p) \in \mathbf{H}_{\bar{u}}^{1}(\Omega) \times$ $\mathbf{H}_{0}(\operatorname{div}, \Omega) \times H^{1}(\Omega)$, which satisfy,

$$
\begin{aligned}
& \int_{\Omega}\left(-\omega^{2} \bar{\rho} \boldsymbol{u}+\mathrm{i} \omega \rho^{f} \boldsymbol{w}\right) \cdot \boldsymbol{v}+\int_{\Omega}[\mathbb{D} \boldsymbol{e}(\boldsymbol{u})-p \boldsymbol{\alpha}]: \boldsymbol{e}(\boldsymbol{v})=\int_{\partial_{\sigma} \Omega} \mathbf{g} \cdot \boldsymbol{v} \\
& \int_{\Omega}\left(-\omega^{2} \rho^{f} \boldsymbol{u}+\mathrm{i} \omega \boldsymbol{\rho} \boldsymbol{w}\right) \cdot \boldsymbol{\psi}+\int_{\Omega}\left[\boldsymbol{K}^{-1} \boldsymbol{w}\right] \cdot \boldsymbol{\psi}+\int_{\Omega} \boldsymbol{\psi} \cdot \nabla p=0 \\
& \mathrm{i} \omega \int_{\Omega} q \boldsymbol{\alpha}: \boldsymbol{e}(\boldsymbol{u})+\int_{\Omega} q \nabla \cdot \boldsymbol{w}+\mathrm{i} \omega \int_{\Omega} \frac{1}{\mu} p q=0
\end{aligned}
$$

for all $(\boldsymbol{v}, \boldsymbol{\psi}, q) \in \mathbf{H}_{0 /}^{1}(\Omega) \times \mathbf{L}^{2}(\Omega) \times L^{2}(\Omega)$. This three field formulation can be converted into a two field formulation in $(\boldsymbol{u}, p)$. From $(2.6)_{2}$ we get $\boldsymbol{w}=-\boldsymbol{H}(\omega)\left(\nabla p-\omega^{2} \rho^{f} \boldsymbol{u}\right)$ for a.a. $x \in \Omega$. On substituting $\boldsymbol{w}$ in the other two equations and assuming more regularity of the test pressure $q \in H^{1}(\Omega)$, obvious integration by parts leads to the following problem for $(\boldsymbol{u}, p) \in \mathbf{H}_{\bar{u}}^{1}(\Omega) \times H^{1}(\Omega)$ satisfying,

$$
\begin{aligned}
& -\omega^{2} \int_{\Omega}\left(\bar{\rho} \mathbf{I}+\mathrm{i} \omega\left(\rho^{f}\right)^{2} \boldsymbol{H}(\omega)\right) \boldsymbol{u} \cdot \boldsymbol{v}+\int_{\Omega}[\mathbb{D} \boldsymbol{e}(\boldsymbol{u})-p \boldsymbol{\alpha}]: \boldsymbol{e}(\boldsymbol{v}) \\
& -\mathrm{i} \omega \int_{\Omega} \rho^{f} \boldsymbol{H}(\omega) \nabla p \cdot \boldsymbol{v}=\int_{\partial_{\sigma} \Omega} \boldsymbol{g} \cdot \boldsymbol{v} \\
& \mathrm{i} \omega \int_{\Omega} q \boldsymbol{\alpha}: \boldsymbol{e}(\boldsymbol{u})-\omega^{2} \int_{\Omega} \rho^{f} \boldsymbol{H}(\omega) \boldsymbol{u} \cdot \nabla q+\int_{\Omega} \boldsymbol{H}(\omega) \nabla p \cdot \nabla q \\
& \quad+\mathrm{i} \omega \int_{\Omega} \frac{1}{\mu} p q=0
\end{aligned}
$$

for all $(\boldsymbol{v}, q) \in \mathbf{H}_{0 /}^{1}(\Omega) \times H^{1}(\Omega)$.

\section{Homogenized model of the double porosity medium}

Since our aim in this paper is to validate the two-scale homogenized model derived in [20], we shall only introduce the resulting equations and problems to be solved in practical simulations. Moreover, we introduce a displacement-velocity formulation for the macroscopic model, which is convenient to solve numerically the wave propagation problem. In this section, we explain how the assumed heterogeneity representing the double porosity material is respected in the context of the asymptotic analysis - this is important for understanding the modelling background of numerical simulations reported in Section 5. Then we introduce the formal asymptotic expansions of the three fields involved in (2.6) and define the so-called local problems for characteristic responses of the representative volume element (RVE). Solutions to these problems are used to compute the homogenized coefficients involved in the macroscopic problem which is presented here in both the weak and strong formulation.

\subsection{Heterogeneity at the mesoscopic level}

There are typically two characteristic lengths: $\ell$ describes the heterogeneity size and $L$ is the relevant macroscopic size. The ration $\varepsilon=\ell / L$ is called the scale parameter. For the "real size" $\operatorname{RVE} Z^{\varepsilon} \subset \mathbb{R}^{3}$, we introduce its rescaled copy $Y=\varepsilon^{-1} Z^{\varepsilon}$ which is called the rescaled elementary cell $Y$ defined by $\left.Y=\prod_{i=k}^{3}\right] 0, a_{k}[$; typically $|Y|=1$, see Fig. 1 . The medium is generated by copies of $Z^{\varepsilon}=\varepsilon Y$ as a periodic lattice, so that $\varepsilon a_{k}$ is the lattice period in the $k$-the coordinate direction. For any given $\varepsilon>0$ we define mesoscopic (zoomed) coordinates $y=\left(y_{k}\right) \in Y$ which for a given "macroscopic" position $x$ are given by the localization function $\mathcal{Y}: x \mapsto y$ defined by: $y_{k}=\mathcal{Y}_{k}(x)=\left(x_{k}-\varepsilon a_{k} \operatorname{int}\left\{x_{k} /\left(\varepsilon a_{k}\right)\right\}\right) / \varepsilon$ for $k=1,2,3$, where int $\{z\}$ denotes the integer part of $z$.

The heterogeneity is described at the mesoscopic scale. The cell $Y$ is decomposed into two disjoint parts $Y_{c}$ and $Y_{m}$, thus, $Y_{c}=Y \backslash \bar{Y}_{m}$, corresponding to the two periodically distributed microporosities which are called the channels and the matrix, respectively. As announced in the introduction, both these subdomains are occupied by the Biot continua, however, they have very different mechanical properties. To take into account large contrasts in the permeability and poroelastic coefficients, $\boldsymbol{\alpha}^{\varepsilon}(x), \mathbb{D}^{\varepsilon}(x)$ and $\boldsymbol{K}^{\varepsilon}(x)$ are defined with respect to the position in the matrix and the channel compartments. The following "scaling ansatz" is considered in the asymptotic analysis of the double porosity medium:

$$
\begin{aligned}
& \mathbb{D}^{\varepsilon}(x)=\chi_{m}(y) \mathbb{D}_{m}(y)+\varepsilon^{2} \chi_{c}(y) \widehat{\mathbb{D}}_{c}(y), \\
& \boldsymbol{\alpha}^{\varepsilon}(x)=\chi_{m}(y) \boldsymbol{\alpha}_{m}(y)+\varepsilon \chi_{c}(y) \boldsymbol{\alpha}_{c}(y), \\
& \boldsymbol{K}^{\varepsilon}(x)=\varepsilon^{2} \chi_{m}(y) \widehat{\boldsymbol{K}}_{m}(y)+\chi_{c}(y) \boldsymbol{K}_{c}(y),
\end{aligned}
$$

where $\chi_{d}$ are the characteristic functions of $Y_{d}, d=m, c$. The other material parameters are expected to attain similar values in both the subdomains, so that,

$$
\begin{aligned}
& \bar{\rho}^{\varepsilon}=\chi_{m}(y) \bar{\rho}_{m}(y)+\chi_{c}(y) \bar{\rho}_{c}(y), \\
& \boldsymbol{\rho}^{\varepsilon}=\chi_{m}(y) \boldsymbol{\rho}_{m}(y)+\chi_{c}(y) \boldsymbol{\rho}_{c}(y), \\
& \mu^{\varepsilon}=\chi_{m}(y) \mu_{m}(y)+\chi_{c}(y) \mu_{c}(y) .
\end{aligned}
$$

Above the rescaled permeability $\widehat{K}_{m}=K_{m}^{\text {phys }} / \varepsilon^{2}$ in the matrix part is obtained using the physical permeability $K_{m}^{\text {phys }}$, so that $\widehat{K}_{m}$ can be computed for a given scale $\varepsilon>0$, when treating a real porous medium. In analogy, the rescaled elasticity $\widehat{\mathbb{D}}_{c}$ and stress coupling coefficients $\boldsymbol{\alpha}_{c}$ can be introduced for given real materials. By virtue of the scaling (3.1), since the elasticity of the upscaled medium depends on the harder phase situated in the matrix $\Omega_{m}$, while the 


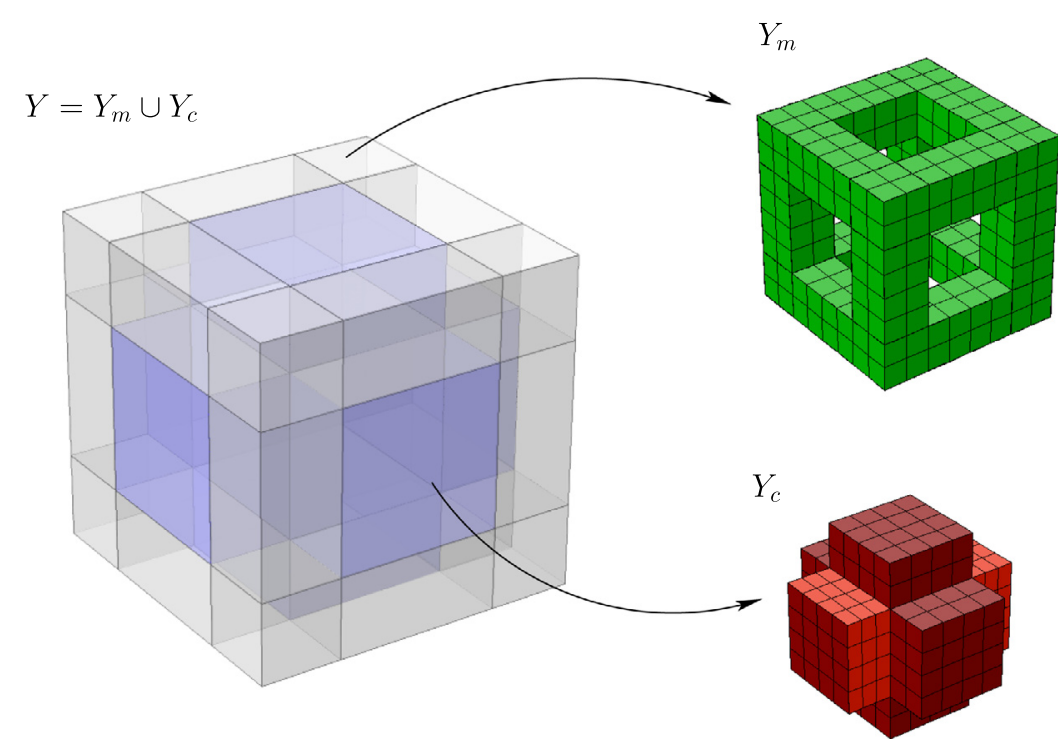

Fig. 1. A RVE with orthogonal connected microstructures. The matrix occupying $Y_{m}$ represents the dual porosity, the channels form the 3D cross, domain $Y_{c}$.

permeability is inherited form the one of the channel part $\Omega_{c}$, it is important that both these components form connected domains. Let us note that in [19], we treated quasistatic loading of similar double porosity media without contrast in the poroelasticity coefficients, so that $\Omega_{m}$ could be constituted by periodically distributed inclusions, cf. [13].

In the asymptotic analysis reported in [20], the scaling employed in (3.1) introduces internal characteristic lengths which are preserved when passing to the limit with $\varepsilon \rightarrow 0$. For modelling real existing materials, the model obtained by homogenization of the porous medium with the heterogeneities according to (3.1) must be interpreted for a fixed scale parameter $\varepsilon_{0}>0$, whereby the following two conditions must be satisfied:

1. The characteristic size $\ell$ of the heterogeneities, i.e. the dimensions of the representative cell $Z^{\varepsilon_{0}}$ must be much smaller than a characteristic macroscopic length $L$ which can be related to the dimensions of a bounded domain $\Omega$, or to the macroscopic wavelength characterizing the material. This gives the "real scale parameter" $\varepsilon_{0}=\ell / L$.

2. For a real structure, the material contrast of the components must correspond to the ansatz (3.1) interpreted with the given small $\varepsilon_{0} \ll 1$. Thus, the real material elasticity in $Y_{c}$ must be much smaller than the one in $Y_{m}$ and in analogy for the other material parameters.

When these two conditions are verified by the real structure of interest, for given physical parameters $K_{m}^{\text {phys }}, \mathbb{D}_{c}^{\text {phys }}$ and $\boldsymbol{\alpha}_{c}^{\text {phys }}$, the reference parameters denoted by $\hat{\cdot}$ are computed using $\varepsilon_{0}$, as follows: $\widehat{K}_{m}=K_{m}^{\text {phys }} / \varepsilon_{0}^{2}, \widehat{\mathbb{D}}_{c}=\mathbb{D}_{c}^{\text {phys }} / \varepsilon_{0}^{2}$, and $\widehat{\boldsymbol{\alpha}}_{c}=\boldsymbol{\alpha}_{c}^{\text {phys }} / \varepsilon_{0}$.

We shall discus these important scaling and contrast aspects in Section 5 .

\subsection{Formal asymptotic expansions}

The macroscopic model has been obtained rigorously using the two-scale convergence method based on the periodic unfolding, see $[20,21]$ for details. Alternatively the formal homogenization approach based on the asymptotic expansion can be used. For this, the convergence result can be interpreted in the sense of the socalled recovery sequences which yield the following first order approximations of the three fields involved in the model:

$$
\begin{aligned}
& u^{\varepsilon}(x)=u^{0}(x)+\chi_{c}(y) \hat{u}(x, y)+\varepsilon \chi_{m}(y) u^{1}(x, y), \\
& w^{\varepsilon}(x)=\chi_{c}(y) \tilde{w}(x, y)+\varepsilon \chi_{m}(y) \hat{w}(x, y), \\
& p^{\varepsilon}(x)=p^{0}(x)+\varepsilon \chi_{c}(y) p^{1}(x, y)+\chi_{m}(y) \hat{p}(x, y) .
\end{aligned}
$$

The macroscopic discharge is given as the mean of $\tilde{w}(x, y)$,

$w^{0}(x)=\int_{Y_{c}} \tilde{w}(x, y) \mathrm{d} y$.

All the two-scale functions of the form $f(x, y)$ are Y-periodic in $y$. Moreover, functions $\hat{u}$ and $\hat{p}$ marked by hat ${ }^{\wedge}$ vanish on the interface $\Gamma$ between the two porosities. Therefore, in the variational formulations, the following function spaces are employed, whereby \# denotes the Y-periodicity of functions,

$\mathbf{H}_{\#, 0}^{1}\left(Y_{c}\right)=\left\{v \in \mathbf{H}_{\#}^{1}\left(Y_{c}\right) \mid v=0\right.$ on $\left.\Gamma\right\}$,
$H_{0, \#}^{1}\left(Y_{m}\right)=\left\{q \in H_{\#}^{1}\left(Y_{m}\right) \mid q=0\right.$ on $\left.\Gamma\right\}$.

In (3.3), it should be noted that the expansion for $w^{\varepsilon}$ at the leading order term $\tilde{w}$ is a two-scale function. This is also the case in the single porosity media, see Proposition 4.1 in [15]. As for $\hat{w}$, there is no explicit condition for $\hat{w}$ on $\Gamma$, while $\tilde{w} \cdot n=0$ on $\Gamma$. It can be seen that this result is independent of the deformation phenomenon and, thus, Theorem 3.2 of [18] applies. A rigorous justification of the truncated expansions (3.3) is reported in [21].

In what follows, we assume a given frequency $\omega$, corresponding to the imposed incident waves, and use the abbreviation $\lambda=\mathrm{i} \omega$. By virtue of the problem linearity and assumed periodicity of the heterogeneous medium, any such $f(x, y)$ can be represented in the decomposed form using the so-called characteristic response functions depending on variable $y$ and using the macroscopic functions depending on $x$ only. Therefore, we consider the two scale functions defined in the matrix part written in the following form:

$$
\begin{aligned}
& u^{1}=\lambda \omega^{k l} e_{k l}^{x}\left(u^{0}\right)+\lambda \omega^{P} p^{0}, \\
& \hat{w}=\lambda \hat{\chi}^{k l} e_{k l}^{x}\left(u^{0}\right)+\lambda \hat{\chi}^{P} p^{0}, \\
& \hat{p}=\lambda \hat{\pi}^{k l} e_{k l}^{x}\left(u^{0}\right)+\lambda \hat{\pi}^{P} p^{0},
\end{aligned}
$$

where all $\omega^{\bullet}, \hat{\chi}^{\bullet}$ and $\hat{\pi}^{\bullet}$ are Y-periodic functions of $y \in Y_{m}$. In analogy, the two scale functions defined in the channel part can be decomposed, as follows: 
$\hat{u}=\lambda \hat{\boldsymbol{\omega}}^{k}\left(\lambda^{2} u_{k}^{0}\right)+\lambda \hat{\boldsymbol{\omega}}^{P} p^{0}+\lambda \hat{\boldsymbol{\omega}}^{\nabla P, k} \partial_{k}^{X} p^{0}$,

$p^{1}=\lambda \pi^{k}\left(\lambda^{2} u_{k}^{0}\right)+\lambda \pi^{P} p^{0}+\lambda \pi^{\nabla P, k} \partial_{k}^{x} p^{0}$,

$\tilde{w}=\lambda \chi^{k}\left(\lambda^{2} u_{k}^{0}\right)+\lambda \chi^{P} p^{0}+\lambda \chi^{\nabla P, k} \partial_{k}^{X} p^{0}$,

where all $\hat{\omega}^{\bullet}, \chi^{\bullet}$ and $\pi^{\bullet}$ are Y-periodic functions of $y \in Y_{c}$. In the next section, we present the local problems for computing all the characteristic responses $\omega, \boldsymbol{\chi}$ and $\pi$ involved in (3.6) and (3.7).

\subsection{Characteristic responses of the mesoscopic RVE}

The responses of the mesoscopic RVE are needed not only to compute the effective medium properties which constitute the macroscopic model of the homogenized medium, but also for reconstruction of the two-scale fields introduced in (3.3) approximating the response of the heterogeneous periodic structure characterized by a given scale parameter $\varepsilon_{0}>0$. We define the following spaces

$\mathcal{A}_{m}=\mathbf{H}_{\#}^{1}\left(Y_{m}\right) \times \mathbf{L}_{\#}^{2}\left(Y_{m}\right) \times H_{0, \#}^{1}\left(Y_{m}\right)$,

$\mathcal{A}_{c}=\mathbf{H}_{\# 0}^{1}\left(Y_{c}\right) \times \mathbf{L}_{\#}^{2}\left(Y_{c}\right) \times H_{\#}^{1}\left(Y_{c}\right)$,

and employ bilinear forms which are now introduced. In domain $Y_{m}$, we shall need

$a_{m}(u, v)=\int_{Y_{m}} \mathbb{D}_{m} e_{y}(u): e_{y}(v), \quad b_{m}(p, v)=\int_{Y_{m}} \boldsymbol{\alpha}_{m}: e_{y}(v) p$,

$d_{m}(p, q)=\int_{Y_{m}} \frac{1}{\mu_{m}} p q, \quad c_{m}(w, z)=\int_{Y_{m}}{\widehat{K_{m}}}^{-1} w \cdot z$,

whereas in $Y_{c}$, we shall use

$a_{c}(u, v)=\int_{Y_{c}} \widehat{\mathbb{D}}_{c} e_{y}(u): e_{y}(v), \quad b_{c}(p, v)=\int_{Y_{c}} \widehat{\boldsymbol{\alpha}}_{c}: e_{y}(v) p$,

$c_{c}(w, z)=\int_{Y_{c}} K_{c}^{-1} w \cdot z$

The characteristic responses $\boldsymbol{\omega}^{\bullet}, \hat{\chi}^{\bullet}$ and $\hat{\pi}^{\bullet}$ defined in $Y_{m}$ are solutions of local problems which have the following generic form:

Find $(\boldsymbol{\omega}, \hat{\boldsymbol{\chi}}, \hat{\pi}) \in \mathcal{A}_{m}$, such that

$a_{m}(\omega, v)-b_{m}(\hat{\pi}, v)=-\frac{1}{\lambda} f_{m}(v)$,

$\left\langle\hat{z}, \nabla_{y} \hat{\pi}\right\rangle_{Y_{m}}+c_{m}(\hat{\chi}, \hat{z})=0$,

$\lambda b_{m}(\hat{q}, \omega)-\left\langle\hat{\boldsymbol{\chi}}, \nabla_{y} \hat{\boldsymbol{q}}\right\rangle_{Y_{m}}+\lambda d_{m}(\hat{\pi}, \hat{q})=-h_{m}(\hat{q})$,

for all $(v, \hat{z}, \hat{q}) \in \mathcal{A}_{m}$, where $f_{m}$ and $h_{m}$ designate a generic form of the right hand side terms of the above problems, see the following table:

\begin{tabular}{lll}
\hline Corrector functions: & Functional $f_{m}(v)$ & Functional $h_{m}(\hat{\boldsymbol{q}})$ \\
\hline$\left(\boldsymbol{\omega}^{k l}, \hat{\boldsymbol{\chi}}^{k l}, \hat{\pi}^{k l}\right)$ & $a_{m}\left(\boldsymbol{\Pi}^{k l}, v\right)$ & $b_{m}\left(\hat{\boldsymbol{q}}, \boldsymbol{\Pi}^{k l}\right)$ \\
$\left(\boldsymbol{\omega}^{P}, \hat{\boldsymbol{\chi}}^{P}, \hat{\pi}^{P}\right)$ & $-b_{m}(1, v)$ & $d_{m}(1, \hat{\boldsymbol{q}})$ \\
\hline
\end{tabular}

We recall that both $\hat{\pi}^{k l}$ and $\hat{\pi}^{P}$ vanish on the interface $\Gamma$.

In the channel part $Y_{c}$, the characteristic responses $\hat{\omega}^{\bullet}, \chi^{\bullet}$ and $\pi^{\bullet}$ satisfy the following generic form of the local problems:

Find $(\hat{\boldsymbol{\omega}}, \boldsymbol{\chi}, \pi) \in \mathcal{A}_{c}$, such that

$a_{c}(\hat{\boldsymbol{\omega}}, \hat{v})+\lambda^{2}\left\langle\bar{\rho}_{c} \hat{\boldsymbol{\omega}}, \hat{v}\right\rangle_{Y_{c}}+\lambda\left\langle\rho^{f} \boldsymbol{\chi}, \hat{v}\right\rangle_{Y_{c}}=-\frac{1}{\lambda} f_{c}(\hat{v})$,

$\lambda^{2}\left\langle\rho^{f} \hat{\boldsymbol{\omega}}, \boldsymbol{\psi}\right\rangle_{Y_{c}}+\lambda\langle\boldsymbol{\rho} \boldsymbol{\chi}, \boldsymbol{\psi}\rangle_{Y_{c}}+c_{c}(\boldsymbol{\chi}, \boldsymbol{\psi})+\left\langle\nabla_{y} \pi, \boldsymbol{\psi}\right\rangle_{Y_{c}}=-\frac{1}{\lambda} g_{c}(\boldsymbol{\psi})$,

$\left\langle\boldsymbol{\chi}, \nabla_{y} q\right\rangle_{Y_{c}}=0$ for all $(\hat{v}, \psi, q) \in \mathcal{A}_{c}$, where $f_{c}$ and $g_{c}$ designate a generic form of the right hand side terms of the above problems, see the following table, where $1_{k}$ is the unit vector of the $k$-th coordinate direction, $\left(1_{k}\right)_{i}=\delta_{i k}$.

\begin{tabular}{lll}
\hline Corrector functions: & Functional $f_{c}(\hat{v})$ & Functional $g_{c}(\boldsymbol{\psi})$ \\
\hline$\left(\hat{\boldsymbol{\omega}}^{k}, \boldsymbol{\chi}^{k}, \pi^{k}\right)$ & $\left\langle\bar{\rho}_{c} 1_{k}, \hat{v}\right\rangle_{Y_{c}}$ & $\left\langle\rho^{f} 1_{k}, \boldsymbol{\psi}\right\rangle_{Y_{c}}$ \\
$\left(\hat{\boldsymbol{\omega}}^{P}, \boldsymbol{\chi}^{P}, \pi^{P}\right)$ & $-b_{c}(1, \hat{v})$ & 0 \\
$\left(\hat{\boldsymbol{\omega}}^{\nabla P, k}, \boldsymbol{\chi}^{\nabla P, k}\right)$ & 0 & $\left\langle 1_{k}, \boldsymbol{\psi}\right\rangle_{Y_{c}}$ \\
\hline
\end{tabular}

We recall that, in this case, all $\hat{\boldsymbol{\omega}}^{k}, \hat{\boldsymbol{\omega}}^{P}$ and $\hat{\boldsymbol{\omega}}^{\nabla P, k}$ vanish on the interface $\Gamma$.

Instead of mixed formulations (3.10) and (3.11), reduced formulations can be considered. From $(3.10)_{2}$, we express $\hat{\boldsymbol{\chi}}=-\widehat{K_{m}} \nabla_{y} \hat{\pi}$, which substituted in the other two equalities yields a problem for $(\omega, \hat{\pi})$ satisfying,

$a_{m}(\omega, v)-b_{m}(\hat{\pi}, v)=-\frac{1}{2} f_{m}(\hat{v})$,

$\lambda b_{m}(\hat{q}, \omega)+\left\langle\widehat{K_{m}} \nabla_{y} \hat{\pi}, \nabla_{y} \hat{q}\right\rangle_{Y_{m}}+\lambda d_{m}(\hat{\pi}, \hat{q})=-h_{m}(\hat{q})$,

for all $(v, \hat{q}) \in \mathbf{H}_{*}^{1}\left(Y_{m}\right) \times H_{0, \#}^{1}\left(Y_{m}\right)$. In analogy, from (3.11) $)_{2}$, upon introducing the mesoscale dynamic permeability $H(\lambda)=[\lambda \boldsymbol{\rho}+$ $\left.K_{c}^{-1}\right]^{-1}$, we express $\boldsymbol{\chi}=-H(\lambda)\left(\nabla_{y} \pi+\lambda^{2} \rho^{f} \hat{\boldsymbol{\omega}}+\lambda^{-1} g_{c}\right)$, where $g_{c}$ is the functional representation associated with $g_{c}(\cdot)$, i.e. $g_{c}(\psi)=\left\langle g_{c}, \psi\right\rangle_{Y_{c}}$. Then, $\boldsymbol{\chi}$ substituted in the other two equalities yields a problem for $(\hat{\boldsymbol{\omega}}, \pi)$ satisfying,

$$
\begin{aligned}
& a_{c}(\hat{\boldsymbol{\omega}}, \hat{v})+\lambda^{2}\left\langle\left[\bar{\rho}_{c}-\lambda\left(\rho^{f}\right)^{2} H(\lambda)\right] \hat{\boldsymbol{\omega}}, \hat{v}\right\rangle_{Y_{c}}-\lambda^{2}\left\langle\rho^{f} H(\lambda) \nabla_{y} \pi, \hat{v}\right\rangle_{Y_{c}} \\
& \quad=\frac{1}{\lambda}\left(\left\langle\rho^{f} H(\lambda) g_{c}, \hat{v}\right\rangle_{Y_{c}}-f_{c}(\hat{v})\right), \\
& \lambda^{2}\left\langle\rho^{f} H(\lambda) \hat{\boldsymbol{\omega}}, \nabla_{y} q\right\rangle_{Y_{c}}+\left\langle H(\lambda) \nabla_{y} \pi, \nabla_{y} q\right\rangle_{Y_{c}}=-\frac{1}{\lambda}\left\langle H(\lambda) g_{c}, \nabla_{y} q\right\rangle_{Y_{c}},
\end{aligned}
$$

for all $(\hat{v}, q) \in \mathbf{H}_{\# 0}^{1}\left(Y_{c}\right) \times H_{\#}^{1}\left(Y_{c}\right)$.

The form of these two local problems (3.12) and (3.13) reveal qualitative differences of the structure response in the two subdomains $Y_{m}$ and $Y_{c}$, representing the dual and the primary porosities. While in $Y_{m}$, the porous medium responses in a regime of quasistatic loading without any inertia effects, these are fully involved in responses of the "softer" material in $Y_{m}$, where the dynamic permeability governs the fluid-structure interaction.

\subsection{Macroscopic model}

Using the classical procedure of the scale separation, the macroscopic equations of the homogenized model are obtained along with the homogenized coefficients which are expressed in terms of the characteristic responses. When boundary tractions are prescribed on $\partial_{\sigma} \Omega$, see $(2.3)$, the limit surface forces $g^{m}$ correspond to the forces acting on the matrix phase, i.e. the stiffer part of the double porous medium which is distributed with a surface volume fraction $\bar{\phi}_{m}$ on $\partial \Omega$. The weak formulation of the macroscopic problem reads, as follows: Find $u^{0} \in \mathbf{H}_{\bar{u}}^{1}(\Omega)$ and $p^{0} \in H_{0}^{1}(\Omega)$ such that

$$
\begin{gathered}
-\omega^{2} \int_{\Omega} \mathcal{M} u^{0} \cdot v^{0}+\int_{\Omega}\left(\mathrm{a} \mathcal{D} e\left(u^{0}\right)-p^{0} \mathcal{B}\right): e\left(v^{0}\right) \\
+\mathrm{i} \omega \int_{\Omega}\left(\mathcal{C} p^{0}-\mathrm{i} \omega \rho^{f} \mathcal{K} \nabla p^{0}\right) \cdot v^{0}=\int_{\partial_{\sigma} \Omega} \phi_{m} g^{m} \cdot v \\
\int_{\Omega} \mathrm{i} \omega q^{0} \mathcal{B}: e\left(u^{0}\right)-\omega^{2} \int_{\Omega} u^{0} \cdot\left(\rho^{f} \mathcal{K} \nabla q^{0}-\underline{\mathcal{C}} q^{0}\right) \\
+\int_{\Omega}\left(\mathcal{K} \nabla p^{0}\right) \cdot \nabla q^{0}+\mathrm{i} \omega \int_{\Omega} \mathcal{H} p^{0} q^{0}=0
\end{gathered}
$$


for all $v^{0} \in \mathbf{H}_{0 /}^{1}(\Omega)$ and $q^{0} \in H_{0}^{1}(\Omega)$. Above, the homogenized coefficients which express the effective medium properties, are computed using solutions of local problems (3.10) and (3.11). The poroelastic coefficients include: $\mathrm{a} \mathbb{D}=\left(\mathcal{D}_{i j k l}\right)$ - the effective elasticity of the drained medium, $\mathcal{B}=\left(\mathcal{B}_{i j}\right)$ - the Biot stress coupling coefficients, and $\mathcal{H}$ - the Biot compressibility; the following expressions can be derived,

$$
\begin{aligned}
& \mathcal{D}_{i j k l}=-\omega^{2} a_{m}\left(\boldsymbol{\omega}^{k l}+\frac{1}{\mathrm{i} \omega} \boldsymbol{\Pi}^{k l}, \boldsymbol{\omega}^{i j}+\frac{1}{\mathrm{i} \omega} \boldsymbol{\Pi}^{i j}\right)+\mathrm{i} \omega c_{m}\left(\hat{\boldsymbol{\chi}}^{i j}, \hat{\boldsymbol{\chi}}^{k l}\right) \\
& \quad-\omega^{2} d_{m}\left(\hat{\pi}^{i j}, \hat{\pi}^{k l}\right), \\
& \mathcal{B}_{i j}= b_{m}\left(1, \boldsymbol{\Pi}^{i j}\right)+\mathrm{i} \omega b_{m}\left(\hat{\pi}^{P}, \boldsymbol{\Pi}^{i j}\right)-\mathrm{i} \omega a_{m}\left(\boldsymbol{\omega}^{P}, \boldsymbol{\Pi}^{i j}\right), \\
& \mathcal{H}=\int_{Y} \mu^{-1}+\mathrm{i} \omega\left(\int_{Y_{m}} \mu_{m}^{-1} \hat{\pi}^{P}+b_{m}\left(1, \omega^{P}\right)+b_{c}\left(1, \hat{\boldsymbol{\omega}}^{P}\right)\right) .
\end{aligned}
$$

The effective dynamic permeability $\mathcal{K}=\left(\mathcal{K}_{i j}\right)$ and other coefficients related to inertia effects are computed, as follows,

$$
\begin{aligned}
& \mathcal{K}_{i j}=\frac{\mathrm{i}}{\omega} \int_{Y_{c}} \chi_{i}^{\nabla P, j}=\mathcal{K}_{j i}, \\
& \mathcal{N}_{i j}=\int_{Y_{c}} \bar{\rho}_{c} \hat{\omega}_{i}^{j}, \\
& \mathcal{M}_{i j}=\int_{Y} \bar{\rho} \delta_{i j}+(\mathrm{i} \omega)^{3} \mathcal{N}_{i j}-\mathrm{i} \omega\left(\rho^{f}\right)^{2} \mathcal{K}_{i j}, \\
& \mathcal{C}_{k}=-\omega^{2} \int_{Y_{c}} \bar{\rho}_{c} \hat{\omega}_{k}^{P}=\omega^{2} b_{c}\left(1, \hat{\boldsymbol{\omega}}^{k}\right) .
\end{aligned}
$$

It is worth noting, that $\mathbb{D}$ and $\mathcal{B}$ depend on the response of the matrix part, i.e. the double porosity represented by $Y_{m}$, while the coefficients in (3.16) are determined by the response in the channels represented by $Y_{c}$.

\subsection{Formulation in $(u, w)$}

From (3.14), we can derive the strong formulation which allows us to reformulate the macroscopic model in terms of the couple $(u, w)$ rather than $(u, \nabla p)$. Thereby we obtain the macroscopic model defined in the form coherent with the original Biot's formulation of the wave propagation in the porous media. Upon integrating by parts in (3.14), these variational equalities yield the following equations to be satisfied in $\Omega$,

$-\omega^{2} \mathcal{M} u^{0}-\nabla \cdot\left(\operatorname{a} \mathbb{D} e\left(u^{0}\right)-\mathcal{B} p^{0}\right)-\mathrm{i} \omega \rho^{f} \mathcal{K} \nabla p^{0}+\mathrm{i} \omega \underline{\mathcal{C}} p^{0}=0$,

$\mathcal{B}: \mathrm{i} \omega e\left(u^{0}\right)+\omega^{2} \rho^{f} \nabla \cdot\left(\mathcal{K} u^{0}\right)+\omega^{2} \underline{\mathcal{C}} \cdot u^{0}-\nabla \cdot\left(\mathcal{K} \nabla p^{0}\right)+\mathrm{i} \omega \mathcal{H} p^{0}=0$,

and the boundary conditions

$u^{0}=\bar{u} \quad$ on $\partial_{u} \Omega$,

$\boldsymbol{\sigma}^{0} \cdot n=\bar{\phi}_{m} g^{m}$ on $\partial_{\sigma} \Omega$,

$w^{0} \cdot n=0$ on $\partial \Omega$,

where $\sigma^{0}$ is the stress and $w^{0}$ is the macroscopic seepage velocity which can be expressed exclusively in terms of the macroscopic permeability,

$\boldsymbol{\sigma}^{0}=\operatorname{a} \mathbb{D} e\left(u^{0}\right)-\mathcal{B} p^{0}$,

$w^{0}=-\mathcal{K}\left(\nabla p^{0}-\omega^{2} \rho^{f} u^{0}\right)$.

By virtue of these macroscopic constitutive equations and using the effective density tensor

$\mathcal{R}=I \int_{Y} \bar{\rho}+(\mathrm{i} \omega)^{3} \mathcal{N}$,

(3.17) can be rewritten, as follows:

$-\omega^{2} \mathcal{R} u^{0}+\mathrm{i} \omega \rho^{f} w^{0}+\mathrm{i} \omega \mathcal{C} p^{0}-\nabla \cdot \sigma^{0}=0$,

$\mathrm{i} \omega \mathcal{B}: e\left(u^{0}\right)+\nabla \cdot w^{0}+\mathrm{i} \omega \mathcal{H} p^{0}+\omega^{2} \underline{\mathcal{C}} \cdot u^{0}=0$.
We shall consider (3.21) 1 and (3.19) 2 in a weak sense; due to the boundary conditions (3.18), obvious integration by parts yields:

$$
\begin{aligned}
& \int_{\Omega}\left(-\omega^{2} \mathcal{R} u^{0}+\mathrm{i} \omega \rho^{f} w^{0}+\mathrm{i} \omega \underline{\mathcal{C}} p^{0}\right) \cdot v+\int_{\Omega} \boldsymbol{\sigma}^{0}: e(v)=\int_{\partial_{\sigma} \Omega} \bar{\phi}_{m} g^{m} \cdot v \\
& \int_{\Omega} p^{0} \nabla \cdot \psi+\int_{\Omega} \omega^{2} \rho^{f} u^{0} \cdot \psi-\int_{\Omega} \psi \cdot \mathcal{K}^{-1} w^{0}=0
\end{aligned}
$$

for all $(v, \psi) \in \mathbf{H}_{0 /}^{1}(\Omega) \times \mathbf{H}_{0}(\operatorname{div}, \Omega)$.

The next step is to express the fluid pressure involved in (3.19) in terms of $\left(u^{0}, w^{0}\right)$; thus, using $(3.21)_{2}$ we get

$$
\begin{aligned}
& p^{0}=-(\mathrm{i} \omega \mathcal{H})^{-1}\left(\mathrm{i} \omega \mathcal{B}: e\left(u^{0}\right)+\nabla \cdot w^{0}+\omega^{2} \underline{\mathcal{C}} \cdot u^{0}\right) \\
& \boldsymbol{\sigma}^{0}=\left(\mathrm{a} \mathcal{D}+\mathcal{H}^{-1} \mathcal{B} \otimes \mathcal{B}\right) e\left(u^{0}\right)-\mathrm{i} \omega \mathcal{H}^{-1} \mathcal{B}\left(\underline{\mathcal{C}} \cdot u^{0}\right) \\
&+\mathcal{H}^{-1}(\mathrm{i} \omega)^{-1} \nabla \cdot w^{0} \mathcal{B} \\
& \boldsymbol{\sigma}^{0}: e(v)+\mathrm{i} \omega p^{0} \underline{\mathcal{C}} \cdot v=e(v):\left(\mathrm{a} \mathcal{D}+\mathcal{H}^{-1} \mathcal{B} \otimes \mathcal{B}\right) e\left(u^{0}\right) \\
& \quad-\omega^{2} \mathcal{H}^{-1} \underline{\mathcal{C}} \otimes \underline{\mathcal{C}}: u^{0} \otimes v \\
&-\mathrm{i} \omega \mathcal{H}^{-1}\left(\left(\underline{\mathcal{C}} \cdot u^{0}\right) \mathcal{B}: e(v)+v \cdot \underline{\mathcal{C}} \mathcal{B}: e\left(u^{0}\right)\right) \\
& \quad+(\mathrm{i} \omega)^{-1} \nabla \cdot w^{0} \mathcal{H}^{-1}(\mathcal{B}: e(v)-\mathrm{i} \omega \underline{\mathcal{C}} \cdot v) .
\end{aligned}
$$

To simplify the notation, we introduce the following tensors $\underline{G}=\left(G_{k i j}\right), a \mathbb{D}^{U}=\left(\mathcal{D}_{i j k l}^{U}\right)$,

$\mathrm{a} \mathbb{D}^{U}=\mathrm{a} \mathbb{D}+\mathcal{H}^{-1} \mathcal{B} \otimes \mathcal{B}, \quad \underline{G}=\underline{\mathcal{C}} \otimes \mathcal{B}$.

It is worth to note, that a $\mathbb{D}^{U}$ depends on $\omega$ mainly due to the sensitivity of $\mathcal{B}$ on $\omega$, while $\mathbb{D}$ is almost insensitive.

The macroscopic problem (3.14) can now be reformulated: Find $\left(u^{0}, w^{0}\right) \in \mathbf{H}_{\bar{u}}^{1}(\Omega) \times \mathbf{H}_{0}(\operatorname{div}, \Omega)$, such that,

$-\omega^{2} \int_{\Omega} v \cdot\left(\mathcal{R}+\mathcal{H}^{-1} \underline{\mathcal{C}} \otimes \underline{\mathcal{C}}\right) u^{0}+\mathrm{i} \omega \int_{\Omega} \rho^{f} w^{0} \cdot v$

$-\mathrm{i} \omega \int_{\Omega}\left(u^{0} \cdot \underline{G}: e(v)+v \cdot \underline{G}: e\left(u^{0}\right)\right)+\int_{\Omega}\left[\mathrm{a} \mathbb{D}^{U} e\left(u^{0}\right)\right]: e(v)$

$+\int_{\Omega}(\mathrm{i} \omega)^{-1} \nabla \cdot w^{0} \mathcal{H}^{-1}(\mathcal{B}: e(v)-\mathrm{i} \omega \underline{\mathcal{C}} \cdot v)=\int_{\partial_{\sigma} \Omega} \bar{\phi}_{m} g^{m} \cdot v$,

$\int_{\Omega} \nabla \cdot \psi \mathcal{H}^{-1}\left(\mathrm{i} \omega \underline{\mathcal{C}} \cdot u^{0}-\mathcal{B}: e\left(u^{0}\right)-(\mathrm{i} \omega)^{-1} \nabla \cdot w^{0}\right)$

$-\int_{\Omega} \psi \cdot\left(\mathcal{K}^{-1} w^{0}-\omega^{2} \rho^{f} u^{0}\right)=0$,

for all $(v, \psi) \in \mathbf{H}_{0 /}^{1}(\Omega) \times \mathbf{H}_{0}(\operatorname{div}, \Omega)$.

Let us now consider a special case when $\underline{\mathcal{C}}=0$, which arises for any mesoscopic structure with constant Biot coefficients in the channel part $Y_{c}$ of the double porous structure, see [20]. Then (3.25) simplifies due to many vanishing terms. The differential form of (3.25) consists of the two differential equations satisfied in $\Omega$,

$$
\begin{aligned}
& -\omega^{2} \mathcal{R} u^{0}+\mathrm{i} \omega \rho^{f} w^{0}-\nabla \cdot\left[\mathrm{a} \mathbb{Z}^{U} e\left(u^{0}\right)+(\mathrm{i} \omega)^{-1} \nabla \cdot w^{0} \mathcal{H}^{-1} \mathcal{B}\right]=0 \\
& -\nabla\left(\mathcal{H}^{-1} \mathcal{B}: e\left(u^{0}\right)+(\mathrm{i} \omega)^{-1} \mathcal{H}^{-1} \nabla \cdot w^{0}\right)-\omega^{2} \rho^{f} u^{0}+\mathcal{K}^{-1} w^{0}=0
\end{aligned}
$$

and taking into account the boundary conditions (3.18).

\section{Reconstruction of the mesoscopic fields}

In this section we provide formulae which enable to reconstruct displacement, pressure and velocity fields at the level of the heterogeneities. This procedure is affected by a given finite scale $\varepsilon_{0}>0$.

\subsection{Folding procedure}

The two-scale field reconstruction is based on the coordinate split related to the periodic lattice. For $\varepsilon_{0}>0$, using the rescaled cell $Z^{\varepsilon_{0}}$ we introduce its local copies $Z^{K, \varepsilon_{0}}$ labeled by index $K$ 
whereby $\left\{\bar{x}^{K}\right\}_{K}$ is the set of centers of each $Z^{K, \varepsilon_{0}}$. For the sake of simplicity, we consider only such domains $\Omega$ which are generated as a union of non overlapping RVEs $Z^{K, \varepsilon_{0}}$, thus (recall that $\bar{Z}$ is the closure of $Z$ )

$\bar{\Omega}=\bar{\cup}_{K \in \Xi_{\Omega}^{\varepsilon_{0}}} Z^{K, \varepsilon_{0}}, \quad Z^{K, \varepsilon_{0}}=Z^{\varepsilon_{0}}+\xi^{K}$,

where $\Xi_{\Omega}^{\varepsilon_{0}}$ is the set of indices $K$ associated to the lattice vector $k=\left(k_{i}\right) \in \mathbb{Z}^{3}$ such that $\xi^{K}=\varepsilon_{0} k_{i} a_{i}$, recalling the definition $\left.Y=\prod_{i}\right] 0, a_{i}[$ and $|Y|=1$.

For any global position $x \in Z^{K, \varepsilon_{0}}$, the local "mesoscopic" coordinate

$y=\left(x-\bar{x}^{K}\right) / \varepsilon_{0}$,

can be introduced, such that $y \in Y$. Then any two-scale function $f(x, y)$ can be evaluated by combining the macroscopic response represented by $\left(u^{0}(x), p^{0}(x)\right)$ with $x \in \Omega$ in our case, and by the local "autonomous" characteristic responses. The folding procedure can be summarized, as follows: for each "real sized" cell $Y^{K, \varepsilon_{0}}$ with its center $\bar{x}^{K}$ evaluate the local responses given below as two-scale functions $f(x, y)$, where $x \in Z^{K, \varepsilon_{0}}$ and $y \in Y$ is given, as described above.

\subsection{Recovery of the local responses}

The homogenization result yields the following recovery formulae for the displacements, pressure and velocity fields. These are expressed as two-scale functions $f(x, y)$ at the global level for $x \in \Omega$, and $y \in Y$ is obtained by (4.2).

In what follows we use the simplified notation: $\lambda=\mathrm{i} \omega$, where $\omega$ is the angular frequency of the incident waves, and $\varepsilon:=\varepsilon_{0}$, the given fixed scale parameter.

- Reconstruction in the subdomain $Y_{m}$ : As the consequence of (3.6) and due to (3.3), the following formulae are obtained which describe the mesoscopic fields for $x \in Y_{m}^{K, \varepsilon_{0}}$, i.e. at the level of the heterogeneity in the "dual porosity" part:

$$
\begin{aligned}
& u^{\varepsilon}(x)=u^{0}(x)+\varepsilon u^{1}(x, y) \\
& =u^{0}(x)+\varepsilon \lambda \omega^{k l}(y) e_{k l}^{x}\left(u^{0}(x)\right)+\varepsilon \lambda \omega^{P}(y) p^{0}(x), \\
& w^{\varepsilon}(x)=\varepsilon \hat{w}(x, y)=\varepsilon \lambda\left(\hat{\chi}^{k l}(y) e_{k l}^{x}\left(u^{0}(x)\right)+\hat{\chi}^{P}(y) p^{0}(x)\right), \\
& p^{\varepsilon}(x)=p^{0}(x)+\hat{p}(x, y)=\left(1+\lambda \hat{\pi}^{P}(y)\right) p^{0}(x)+\lambda \hat{\pi}^{k l}(y) e_{k l}^{x}\left(u^{0}(x)\right) \text {. }
\end{aligned}
$$

- Reconstruction in the subdomain $Y_{c}$ :

As the consequence of (3.7) and due to (3.3), the following formulae are obtained which describe the mesoscopic fields for $x \in Y_{c}^{K, \varepsilon_{0}}$, i.e. at the level of the heterogeneity in the "primary porosity" part:

$$
\begin{aligned}
u^{\varepsilon}(x) & =u^{0}(x)+\hat{u}(x, y) \\
= & \left(1+\lambda^{3} \hat{\boldsymbol{\omega}}^{k}(y)\right) u^{0}(x)+\lambda\left(\hat{\boldsymbol{\omega}}^{P}(y) p^{0}(x)+\hat{\boldsymbol{\omega}}^{\nabla P, k}(y) \partial_{k}^{x} p^{0}(x)\right), \\
w^{\varepsilon}(x) & =w(x, y)=\lambda\left(\boldsymbol{\chi}^{k}(y) \lambda^{2} u_{k}^{0}(x)+\boldsymbol{\chi}^{P}(y) p^{0}(x)+\chi^{\nabla P, k}(y) \partial_{k}^{x} p^{0}(x)\right), \\
p^{\varepsilon}(x) & =p^{0}(x)+\varepsilon p^{1}(x, y) \\
= & \left(1+\varepsilon \lambda \pi^{P}(y) p^{0}(x)\right)+\lambda \varepsilon\left(\pi^{k}(y) \lambda^{2} u_{k}^{0}(x)+\pi^{\nabla P, k}(y) \partial_{k}^{x} p^{0}(x)\right) .
\end{aligned}
$$

It is worth noting that $p^{1}$ and thereby the corrector functions $\pi^{P}, \pi^{\nabla P, k}$, and $\pi^{k}$ are not needed to compute the effective properties of the homogenized medium, as pointed out in paper [20]. However, the fluctuations represented by the two-scale function $p^{1}(x, y)$ are involved when reconstructing the solutions for a given $\varepsilon_{0}$.
Remark 1 Using the RVE $Z^{\varepsilon_{0}}$ instead of the unit cell $Y$ for computing the characteristic responses. In practical computations, the real sized cell $Z^{\varepsilon_{0}}$ can be used to solve the local problems (3.10) and (3.11), and to compute the homogenized coefficients, however, the following rules must be respected:

(i) The physical material properties (the coefficients $K_{m}^{\text {phys }}, \mathbb{D}_{c}^{\text {phys }}$ and $\boldsymbol{\alpha}_{c}^{\text {phys }}$ ) are plugged into the bilinear forms $c_{m}(\cdot, \cdot), a_{c}(\cdot, \cdot)$ and $b_{c}(\cdot, \cdot)$ to replace the rescaled coefficients $\widehat{K_{m}}, \widehat{\mathbb{D}_{c}}$, and $\widehat{\boldsymbol{\alpha}_{c}}$.

(ii) The characteristic responses computed for such modified problems are different from those computed in the rescaled cell $Y$, thus according to (3.10) and (3.11). Nevertheless, the correct homogenized coefficients are computed with such modified characteristic responses using the expressions (3.15) and (3.16), where the physical material properties are employed, as in the local problems, and $Y$ is replaced by $Z^{\varepsilon_{0}}$, however multiplication by $\left|Z^{\varepsilon_{0}}\right|^{-1}$ must be applied in all the expressions which are presented for the unit cell, $|Y|=1$.

Then, the reconstruction of the solutions with the characteristic responses computed on $Z^{\varepsilon_{0}}$ is done formally according to (4.3) and (4.4), where in all expressions we put $\varepsilon=1$.

The above assertions can easily be seen by a simple rescaling, i.e. using $x=\varepsilon_{0} y$ when transforming all expressions form domain $Y$ to $Z^{\varepsilon_{0}}$. It also clarifies the role of the scaling ansatz in the asymptotic behaviour of the "large contrast" materials: by a proper scaling of the material parameters, an internal scale is introduced which is retained in the limit as $\varepsilon$ tends to 0 .

\section{Numerical tests}

In this section, we study how the approximation quality of the homogenized model is influenced by the material contrast. The aim is twofold. First we illustrate how the macroscopic solution reconstruction enables to capture some local effects related to the wave propagation at the mesoscopic scale, where the strong heterogeneity is introduced in the periodic material coefficients. Second, while changing the material contrast in the sense of the scaling ansatz (3.1), but modified according to (5.1), as explained below, we compare responses of three models:

1. The model of the heterogeneous FSPM generated as the periodic medium with a given fixed size of the heterogeneity period $\ell_{0}$ and constituted by two different materials. The solutions are obtained using the finite element (FE) method upon discretization of the weak formulations.

2. The double porosity homogenized model (3.17) with the homogenized coefficients computed by (3.15) and (3.16).

3. The single porosity homogenized model derived in [15], where the equations are also given by (3.17), but the homogenized coefficients are computed by using the formulae reported in Appendix A, see (7.6).

\subsection{Heterogeneity and material properties}

We consider a periodically heterogeneous material with the characteristic size $\ell_{0}=\varepsilon_{0} L$, where $L$ is a given macroscopic length which can be related to the macroscopic wavelength of propagating waves. Let us consider a reference isotropic poroelastic material described in terms of the mesoscopic mechanical properties introduced in Table 1 . The following parameters are defined by piecewise constant functions, 
Table 1

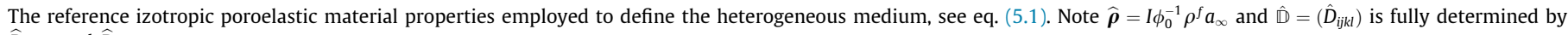
$\widehat{D}_{1111}$ and $\widehat{D}_{1212}$.

\begin{tabular}{|c|c|c|c|c|c|c|c|c|}
\hline $\begin{array}{l}\phi_{0} \\
(-)\end{array}$ & $\begin{array}{c}\rho^{s} \\
\left(\mathrm{~kg} / \mathrm{m}^{3}\right) \\
\end{array}$ & $\begin{array}{c}\rho^{f} \\
\left(\mathrm{~kg} / \mathrm{m}^{3}\right) \\
\end{array}$ & $\begin{array}{l}\widehat{D}_{1111} \\
(\mathrm{GPa}) \\
\end{array}$ & $\begin{array}{l}\widehat{D}_{1212} \\
(\mathrm{GPa}) \\
\end{array}$ & $\begin{array}{c}\widehat{\boldsymbol{\alpha}}=\hat{\alpha} \mathbf{I} \\
(-)\end{array}$ & $\begin{array}{c}\hat{\mu} \\
(\mathrm{GPa}) \\
\end{array}$ & $\begin{array}{c}\hat{K}=\hat{\kappa} \mathbf{I} \\
\left(\mathrm{m}^{2} /\left(\mathrm{Pa} \mathrm{s}^{-1}\right)\right)\end{array}$ & $\begin{array}{l}a_{\infty} \\
(-)\end{array}$ \\
\hline 0.3 & 2650 & 1000 & 16.92 & 7.7 & 0.825 & 7.473 & $1 \times 10^{-9}$ & 1 \\
\hline
\end{tabular}

$\bar{\rho}^{\varepsilon_{0}}(x)=\bar{\rho}, \quad \mathbb{D}_{0}^{\varepsilon}(x)=\chi_{m}(y) \widehat{\mathbb{D}}+r^{2}\left(\varepsilon_{0}\right) \chi_{c}(y) \widehat{\mathbb{D}}$,

$\boldsymbol{\rho}^{\varepsilon_{0}}(x)=\widehat{\boldsymbol{\rho}}, \quad \boldsymbol{\alpha}_{0}^{\varepsilon}(x)=\chi_{m}(y) \widehat{\boldsymbol{\alpha}}+r\left(\varepsilon_{0}\right) \chi_{c}(y) \widehat{\boldsymbol{\alpha}}$,

$\mu^{\varepsilon_{0}}(x)=\widehat{\mu}, \quad K_{0}^{\varepsilon}(x)=r^{2}\left(\varepsilon_{0}\right) \chi_{m}(y) \widehat{K}+\chi_{c}(y) \widehat{K}$,

where $y \in Y$ is associated to a macroscopic coordinate $x$ by the formula (4.2) and by $r\left(\varepsilon_{0}\right)$ we denote the contrast between the corresponding material properties in the two phases. The densities $\bar{\rho}$ and $\widehat{\boldsymbol{\rho}}$ are given by $\bar{\rho}=\left(1-\phi_{0}\right) \rho^{s}+\phi_{0} \rho^{f}$ and $\widehat{\boldsymbol{\rho}}=I \phi_{0}^{-1} \rho^{f} a_{\infty}$, respectively. Although the homogenized model of the double porosity medium is derived for $\varepsilon \rightarrow 0$ using $r(\varepsilon)=\varepsilon$, see (3.1), the obtained "limit model" can be interpreted independently of $\varepsilon_{0}$. In principle, if a "large contrast" - the double porosity (DP) medium is considered, the material properties should be such that,

(i) the parameter $r$ is small enough,

(ii) the scale $\varepsilon_{0}=\ell_{0} / L$ is small enough.

By the "low contrast" - single porosity (SP) medium, we mean such a FSPM, where the contrast parameter is closed to one, thus $r \approx 1$. For this situation, the homogenized model was derived in [15] with material properties being independent of $\varepsilon$, so in (3.1) one puts $\varepsilon=1$. It is worth to note that, if one material is used for both components of the medium represented by subdomains $Y_{m}$ and $Y_{c}$, of the unit cell, thus for $r=1$ in (5.1), the homogeneous medium is recovered by the single porosity (SP) model. The DP model cannot describe such a situation, since the model systematically "neglects" the elasticity of the channels and the permeability of the matrix parts independently of $r$. Therefore, if $r \approx 1$, it provides an extremely poor approximation of the real material behaviour.

It this study, we confine to low frequency bands (up to $\approx 10^{4} \mathrm{rad} / \mathrm{s}$ ), so that no correction of the static permeability with respect to the frequency is needed. With this restriction and by virtue of the considered type of the material heterogeneity (the same fluid, microscopic volume fractions, and tortuosities in both the subdomains) the density $\widehat{\rho}$ is homogeneous at the mesoscopic level, as defined above.

\subsection{Geometry and FE models}

Domain $\Omega$ has the form of a bar generated according to (4.1), where $\xi^{K}=\left(\xi_{1}^{K}, 0,0\right)$ and $\xi_{1}^{K}=K \varepsilon_{0} a_{1}, K=0, \ldots, 49$, thus, using 50 copies of the RVE $Z^{\varepsilon_{0}}$ illustrated in Figs. 2(a) and 3. While both the microporosities are characterized by the porosity $\phi_{0}=0.3$ describing the $30 \%$ fraction of the fluid, the mesoscopic volume fraction is $\left|Y_{c}\right| /|Y|=0.5$. The RVE is formed as a parallelepiped (a rectangular cuboid), with dimensions $0.01 \times 0.001 \times 0.01 \mathrm{~m}$, so that $a_{1}=a_{3}=1, a_{2}=0.1$, whereby $\varepsilon_{0}=0.02$, see Section 3.1 . Therefore, $\ell_{0}=0.01 \mathrm{~m}$, which yields the relevant macroscopic length $L=0.5 \mathrm{~m}$ denoting the "length" of the column $\Omega$. In the context of the wave propagation problem, the macroscopic wavelength $\Lambda$ should be in the range of $L$, but in any case such that $\Lambda>10 \ell_{0}=10 \varepsilon_{0} L$. We remark, that the cell $Y$, i.e. the rescaled RVE, has non-unit volume, so that $|Y| \neq 1$. As the consequence, in definitions of the homogenized coefficients, all integrals over $Y_{m}$, or $Y_{c}$ must be understood in the sense of the volume average $|Y|^{-1} \int_{Y_{d}}, d=m, c$.

The FE method was used to compute numerical solutions for all three problems involved in our study. The FE mesh of $\Omega$ is obviously generated using the mesh of the RVE $Z^{\varepsilon_{0}}$. Since the model equations of the heterogeneous medium and both the homogenized media retain the same structures, see (3.26), the FE (macroscopic) models are derived from the weak formulation (3.25), whereby the following modifications are made:

- The heterogeneous medium. The material parameters are defined in $Z^{\varepsilon_{0}}$ according to (5.1). In (3.26), $\mathcal{R}$ is substituted by $\bar{\rho} I$, tensors a $\mathbb{D}, \mathcal{B}$, and $\mathcal{K}$ are substituted by $\mathbb{D}, \boldsymbol{\alpha}$, and $K$, thus the following replacements are used: (a)

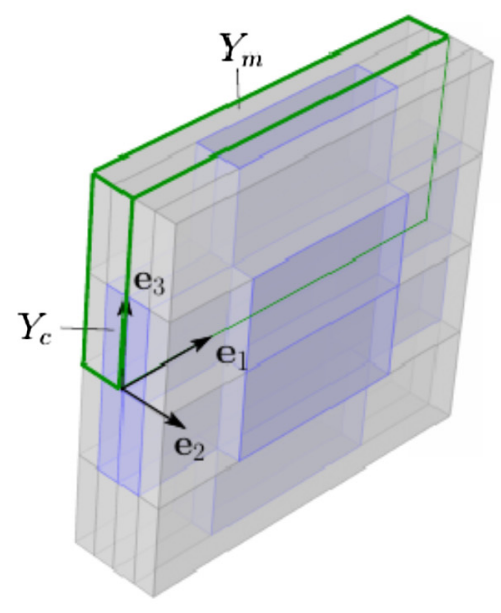

(b)

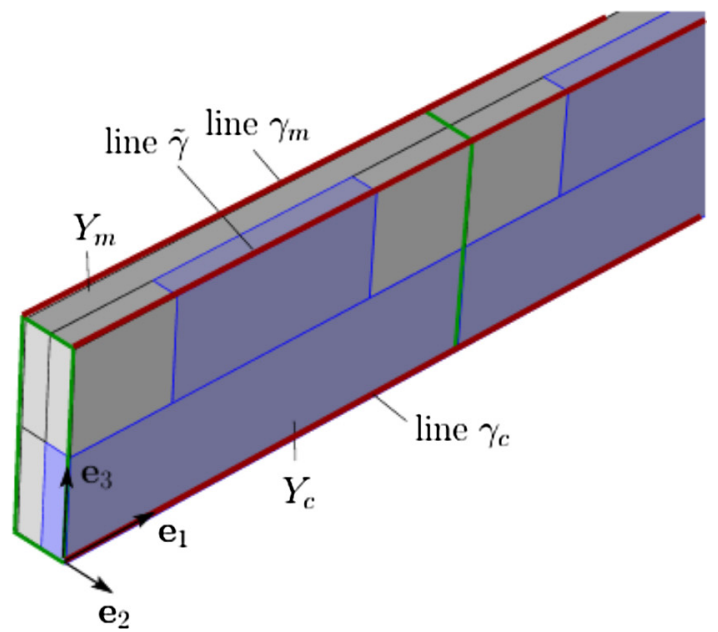

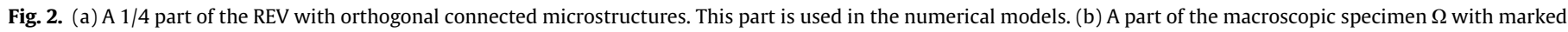
positions of the three lines $\gamma_{c}, \gamma_{m}$ and $\tilde{\gamma}$ along which the displacement amplitudes $u_{1}$ are displayed. 
$\mathrm{a} \mathbb{D}^{U}:=\mathbb{D}+\mu \boldsymbol{\alpha} \otimes \boldsymbol{\alpha}, \quad \mathcal{K}^{-1}:=\mathrm{i} \omega \boldsymbol{\rho}+K^{-1}$.

- The simple porosity medium (SP model). In (3.26), replacements of the corresponding coefficients defined in Appendix A are obvious.

In a more general situations, although coefficients $\underline{\mathcal{C}}$ and $\underline{G}$ are involved in the double porosity model, see (3.25), their analogs exist neither in the SP model, nor in the model of the heterogeneous medium.

\subsection{Boundary conditions}

The boundary $\partial \Omega$ is decomposed into the disjoint parts $\Gamma_{0}, \Gamma_{L}$ and $\partial_{S} \Omega$, such that $\partial_{S} \Omega=\partial \Omega \backslash\left(\Gamma_{0} \cup \Gamma_{L}\right)$, where $\Gamma_{0}=\left\{x \in \partial \Omega: x_{1}=0\right\}$ and $\Gamma_{L}=\left\{x \in \partial \Omega: x_{1}=L\right\}$. We apply boundary conditions to simulate a plane wave propagating in the direction $e_{1}$ in an infinite medium situated in the half-space $x_{1}>0$. Therefore, on $\Gamma_{0}$, the displacements normal to the surface are prescribed, i.e. $\left.u \cdot n\right|_{\Gamma_{0}}=\bar{U}$ being a given scalar constant. If this condition is applied on the boundary of a homogeneous medium, the wave response must be independent of the two transverse coordinates $x_{2}$ and $x_{3}$. In our special periodic heterogeneous medium, due to the symmetry of the geometry, the considered isotropic mesoscopic material distributed using a characteristic functions $\chi_{d}, d=m, c$, see (5.1), and due to the imposed incident plane wave represented by $\bar{U}$, the wave response is symmetric with respect to the planes of symmetries. Therefore, the following boundary conditions are applied:

$$
\begin{aligned}
& u \cdot n=\bar{U} \quad \text { on } \quad \Gamma_{0}, \\
& u \cdot n=0 \quad \text { on } \partial_{S} \Omega \cup \Gamma_{L}, \\
& \boldsymbol{\sigma}: n \otimes t=0 \quad \text { on } \partial \Omega, \\
& w \cdot n=0 \quad \text { on } \partial \Omega,
\end{aligned}
$$

where $n$ is the unit normal vector outward to $\Omega$, while $t$ is any unit tangent vector of the surface. Moreover, due to the above mentioned symmetry of the RVE with respect to the three orthogonal planes aligned with the coordinates, the computational model can be created with only $1 / 4$ of the domain, see Fig. 2(a).

\subsection{Influence of the contrast parameter $r$}

Since the aim of the presented work is to validate the two-scale model of the homogenized FSPM featured by the large contrast, the numerical tests were motivated by two questions:

1. What is the sufficient contrast $r_{0}<1$, such that for $r<r_{0}$ the double porosity model approximates the heterogeneous medium better, than the single porosity (low contrast) model?

2. How the reconstructed solution, as described in Section 4, can approximate the local effects which can be observed at the level of heterogeneities?

To reply on these questions, for a fixed frequency $\omega=5 \times 10^{4} \mathrm{rad} / \mathrm{s}$, a number of simulations have to be done while changing the contrast $r$, thereby the material properties of the two microporous materials according to (5.1). It is to be remarked, that $r$ is changed without modifying the heterogeneity size, i.e. $\varepsilon_{0}$ is fixed, while the model convergence is related to sequences of solutions parameterized by $\varepsilon$, so that simultaneously $r \equiv \varepsilon \rightarrow 0$. As the result, internal lengths are introduced in the limit model: one related to the flows in the dual porosity $Y_{m}$, the other related to the characteristic wavelength of the very compliant poroelastic material in channel $Y_{c}$. In our test, however, these internal lengths change with $r$.
In the computations, we considered contrast $r \in\{1,0.1,0.01,0.0032\}$. The choice of $r=0.0032$ was a compromise between the desired value 0.001 and limitations of the numerical solutions requiring very fine meshes to resolve the "direct" problem described by the RM model in the entire domain $\Omega$, see Fig. 3 (bottom). For the contrast $r=0.1$, the number of DOFs was $2,345,004$, while for contrast $r \leqslant 0.01$, the number of DOFs was $13,425,882$, thus, reaching our limits concerning the computational power available. It is worth to emphasize, that the DP and SP models of the homogenized medium require a fine mesh to discretize the RVE, while relatively rough discretization is sufficient to discretize the macroscopic domain $\Omega$.

The results are reported in Figs. 4-13, where we adhere the following style. The coordinate $x_{1} \in[0,0.5] \mathrm{m}$ spans the whole length $L=0.5 \mathrm{~m}$ of the specimen consisting of 50 copies of the RVE. In all figures we display three solutions of the displacement field, namely the $u_{1}$ component, obtained by the three models: FE model of the heterogeneous medium, or "the reference medium model" (RM), a model of the single porosity (SP) medium, and a model of the double porosity (DP) medium. For the SP and DP models, the macroscopic response $u_{1}^{0}$ is displayed. Moreover, for the DP model which is in our focus, the reconstructed response $u_{1}^{\varepsilon_{0}}$ defined according to (4.3) and (4.4) is depicted. The curves are traced along three different lines, all aligned with the $e_{1}$ axis, see Fig. 1(b). Line $\gamma_{m}$ passes through the dual porosity (the matrix) only, line $\gamma_{c}$ passes through the primary porosity (the channels) only, whereas line $\tilde{\gamma}$ passes through both the phases (the channels and the matrix).

As expected, the DP model provides a good approximation of the RM solutions for small values of $r$, i.e. when the contrast is relatively large, while the SP model is suitable for media, where the two materials are comparable. We shall now discus the particular cases of $r$. By the macroscopic wavelength we mean the length of waves $u_{1}\left(x_{1}, x^{\prime}\right)$ on interval $\left.x_{1} \in\right] 0, L[$ for a given transverse position $x^{\prime}=\left(x_{2}, x_{3}\right)$ computed for the imposed frequency $\omega$ and the boundary conditions, i.e. $u_{1}\left(0, x^{\prime}\right)=1$ and $u_{1}\left(L, x^{\prime}\right)=0$. The transverse position $x^{\prime}$ corresponds to one of the three lines $\gamma_{c}, \gamma_{m}$, or $\tilde{\gamma}$. Therefore, in figures, we use notation $u_{1}(x), x \in \gamma$ to refer to the trace of $u_{1}$ on the particular line. By $u_{1}^{\mathrm{cr}}(x)=u_{1}^{\varepsilon_{0}}(x)$ we shall refer to the reconstructed displacement response of the DP model, see Eqs. (4.3) and (4.4) and Remark 1.

Homogeneous material, $r=1$, see Fig. 4. The SP homogenized model describes also the homogeneous medium, thus, the solutions of the RM and SP models are identical. In principle, the DP model cannot describe a homogeneous medium and, thus, provides a completely wrong response. Since the DP model underestimates the elasticity and the permeability, the imposed frequency $\omega$ induces higher modes than for the real material which is stiffer, so that $\omega$ corresponds to lower modes.

Very weak contrast, $r=0.1$, see Figs. 5-7. In general, the SP model solution matches very well with the RM solution. In particular, both the wavelength and the amplitude are captured. It can be noticed, that the local undulations captured by the RM solutions are negligible (no corrector-based reconstruction was applied for the SP model). The DP model still underestimates the elasticity of the weak material phase in the channels, i.e. on $\gamma_{c}$, see Fig. 6, therefore the local undulations captured by the reconstructed solution $u_{1}^{\mathrm{cr}}(x), x \in \gamma_{c}$ are remarkable. Note that for the RM and SP models, there is not much difference between the responses on $\gamma_{c}$ and $\gamma_{m}$.

Moderate contrast, $r=0.01$, see Figs. $8-10$. In the stiffer part, i.e. on $\gamma_{m}$, the three models are comparable, although the wavelength of the RM response is better approximated by the SP model, see Fig. 10. However, the reconstructed solution $u_{1}^{\text {cr }}$ of 

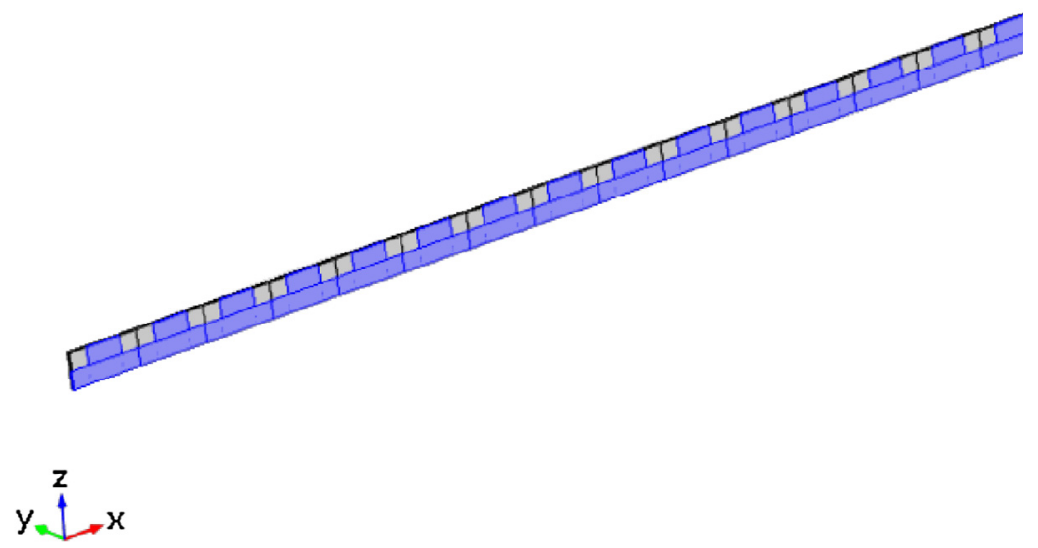

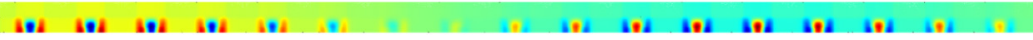

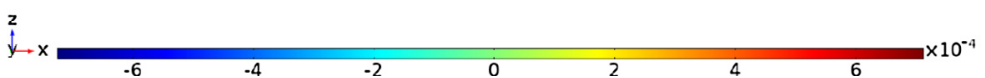

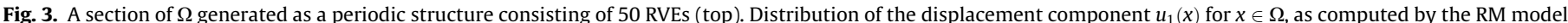

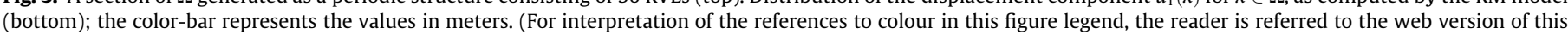
article.)

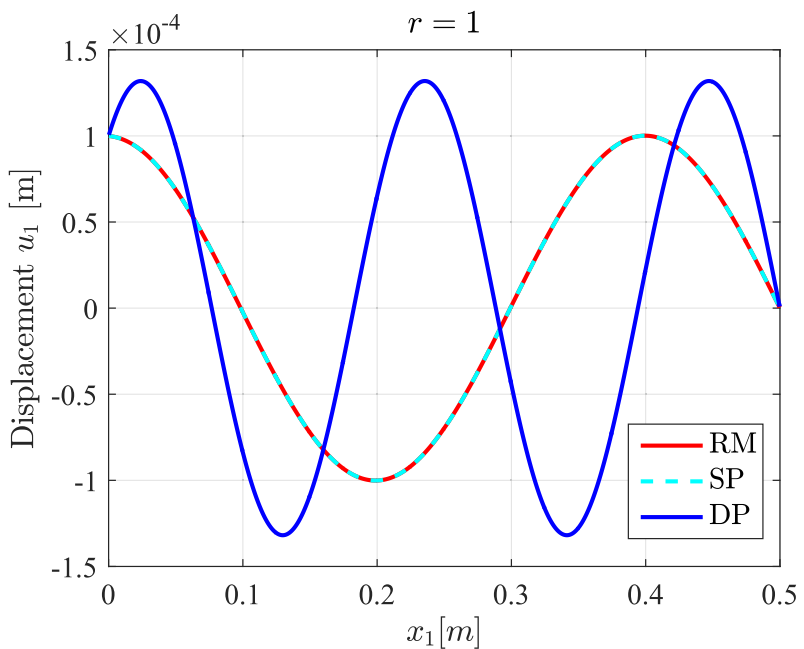

Fig. 4. Solutions of $u_{1}(x), x \in \gamma$, in a homogeneous domain; the SP model matches perfectly with the RM model. The coordinate $x_{1} \in[0,0.5] \mathrm{m}$ spans the whole length $L=0.5 \mathrm{~m}$ of the specimen consisting of 50 copies of the RVE, see Section 5.2.

the DP model captures the local undulations; it is worth to note, that the amplitudes on $\gamma_{c}$ are about four times higher that those on $\tilde{\gamma}$. There is also a remarkable attenuation of the propagating waves in the weaker material; for the DP model, the amplitudes $u_{1}(x)$ are getting smaller with increasing distance $\left.x_{1} \in\right] 0, L[$. Large contrast, $r=0.0032$, see Figs. 11-13. This contrast is already sufficient to show, that the DP model gives a very good approximation of the reference solution computed using the RM model, although for the latter, the numerical approximation becomes an issue, perhaps even more refined mesh would be needed to respect higher modes propagating in the soft mate- rial. Nevertheless, the SP model fails totally to provide a reasonable estimation of the macroscopic wavelength. Both the DP and RM models indicate attenuation effects in the sense explained above. These are associated with the flows in the dual porosity, i.e. in the matrix part, so that they are visible not only in Figs. 11 and 12, but also on responses of the stiffer phase, the lines $\gamma_{m}$, see Fig. 13 .

In a summary, the reported tests demonstrate usefulness of the DP model. It appears that for the contrast $r \approx 0.003$ or smaller, the DP models describes correctly important features of the propagating waves, namely the wavelength; while the solution using the SP model indicate the wavelength $\Lambda_{S P M} \approx 0.22 \mathrm{~m}$ almost independently of the contrast parameter (but for $r \leqslant 0.1$ ), the wavelength $\Lambda_{D P M}$ estimated using the DP model varies; while $\Lambda_{D P M}^{r=0.1} \approx 0.214 \mathrm{~m}$ for $r=0.1$, it increases with $r \rightarrow 0$, so that $\Lambda_{D P M}^{r=0.0032} \approx 0.4 \mathrm{~m}$. As an important aspect, correcting the macroscopic response of the DP model using the procedure described in Section 4 is an indispensable part of the computation, as can be see in Figs. 8 and 9 and Figs. 11 and 12. Finally, comparing the wavelengths $\Lambda \in] 0.2,0.4[\mathrm{~m}$ with the heterogeneity size $\ell_{0}=0.01 \mathrm{~m}$, we obtain an estimate of the scale parameter $\varepsilon_{0}$, thus $\varepsilon_{0} \approx \ell_{0} / \max \{\Lambda\}=0.01 / 0.4=0.025$. On one hand, by virtue of the scaling ansatz (3.1) employed in the asymptotic analysis, see this estimate reveals that the minimum feasible contrast should be $r<0.025$. On the other hand, we observed, that the contrast should be much higher, thus $r<0.003$ to have a good approximation.

\section{Conclusion}

We discussed computational modelling of waves propagating in a double porous fluid-saturated medium. The macroscopic 

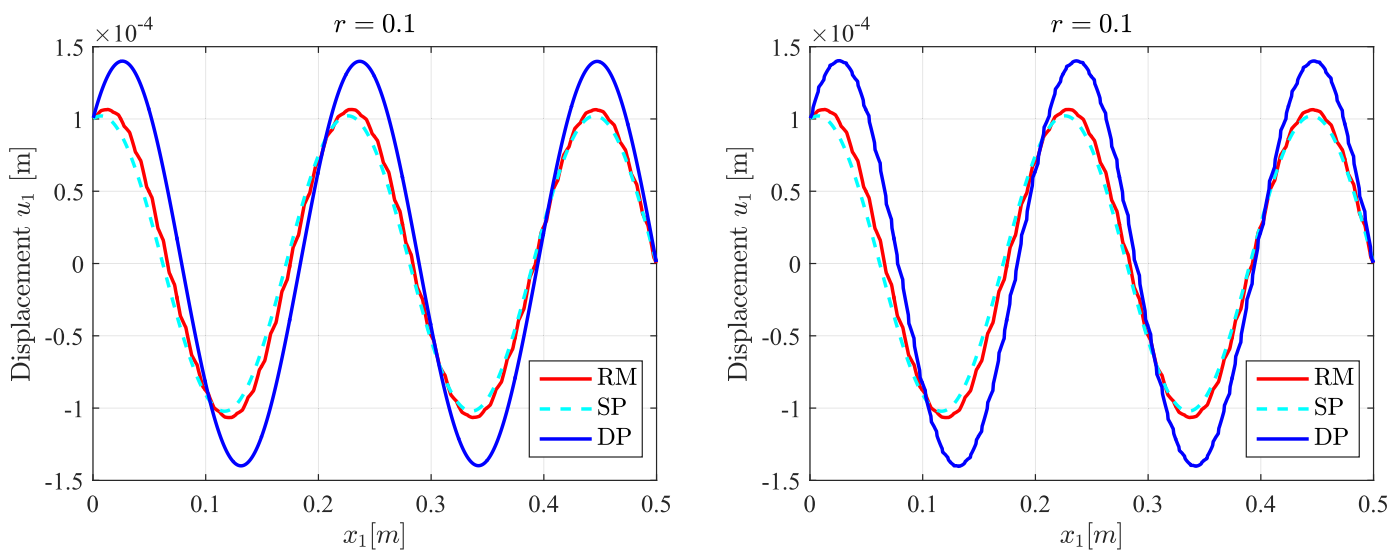

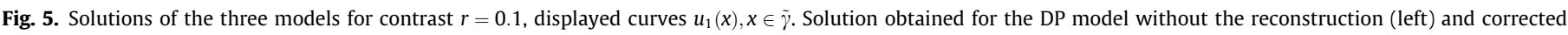
solution $u_{1}^{\text {cr }}$ obtained by the DP model with reconstruction (right).
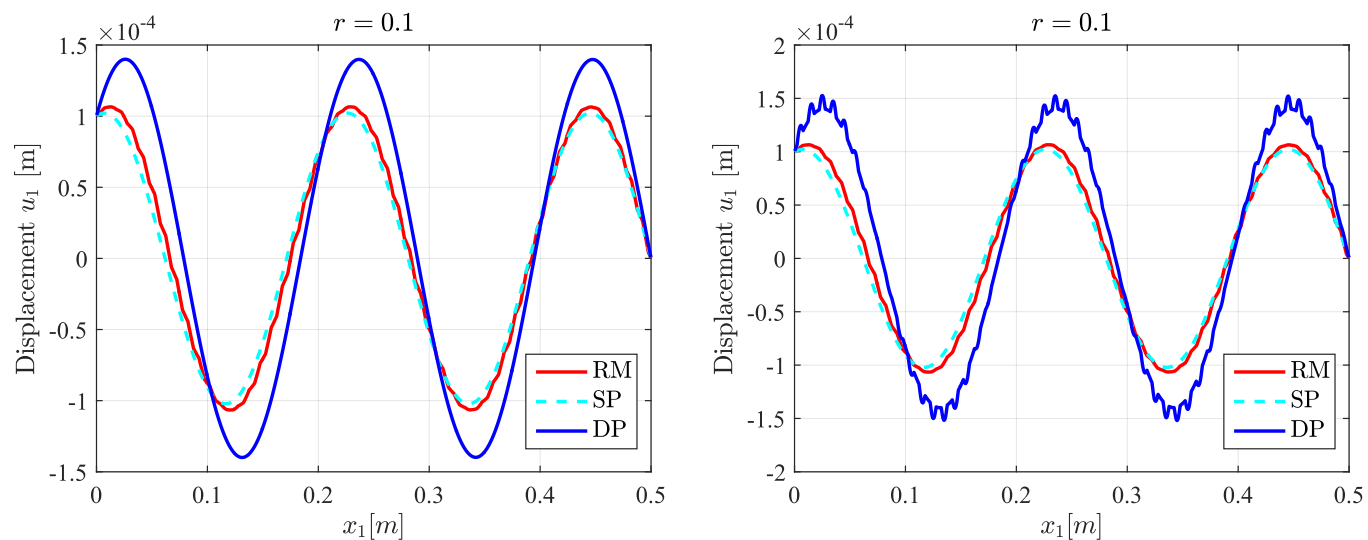

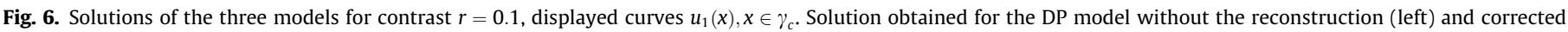
solution $u_{1}^{\mathrm{cr}}$ obtained by the DP model with reconstruction (right).
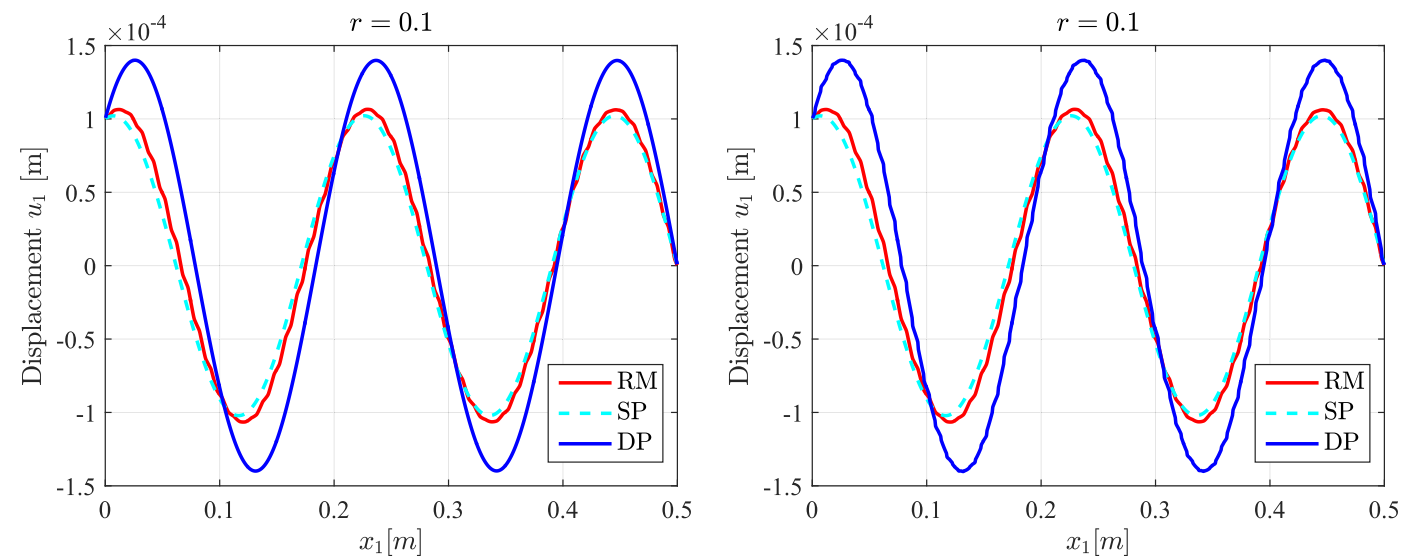

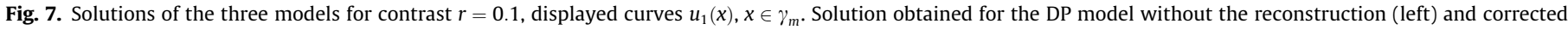
solution $u_{1}^{\mathrm{cr}}$ obtained by the DP model with reconstruction (right).

effective model of this double porosity (DP) medium was derived by the homogenization with the large contrast ansatz which modifies the homogenization result, see [20], when compared with the single-porosity (SP) model of the same type of the medium, but without the scaling ansatz, see Mielke and Rohan [15]. In the DP model, all the effective material properties depend on the frequency of incident waves and a new vectorial coefficient appears which is involved in the stress coupling operator.
The homogenized properties are evaluated using characteristic responses of the representative elementary volume. These responses are governed by local problems which for the DP model have more complex structures than those involved in the SP model. In particular, the deformation phenomena are involved in the local problem determining the dynamic permeability of the DP model.

In this paper, we aimed to validate the DP model and compare it with the SP model which is intended for moderately 

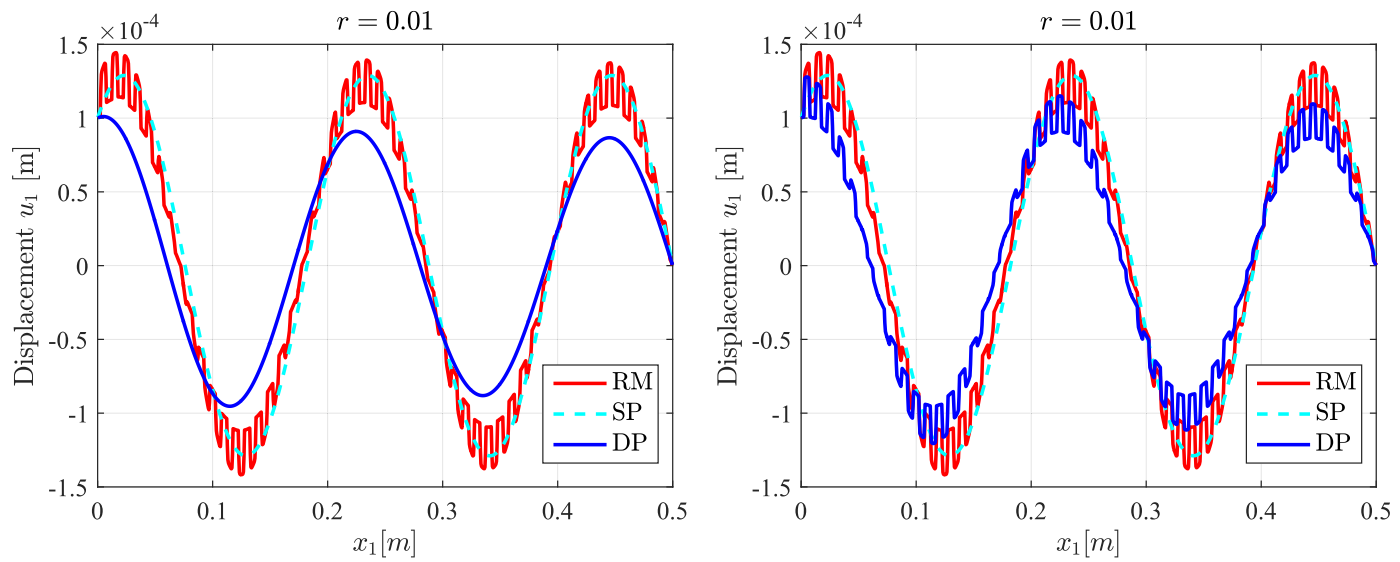

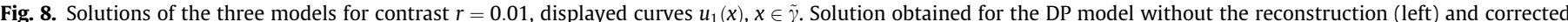
solution $u_{1}^{\mathrm{cr}}$ obtained by the DP model with reconstruction (right).
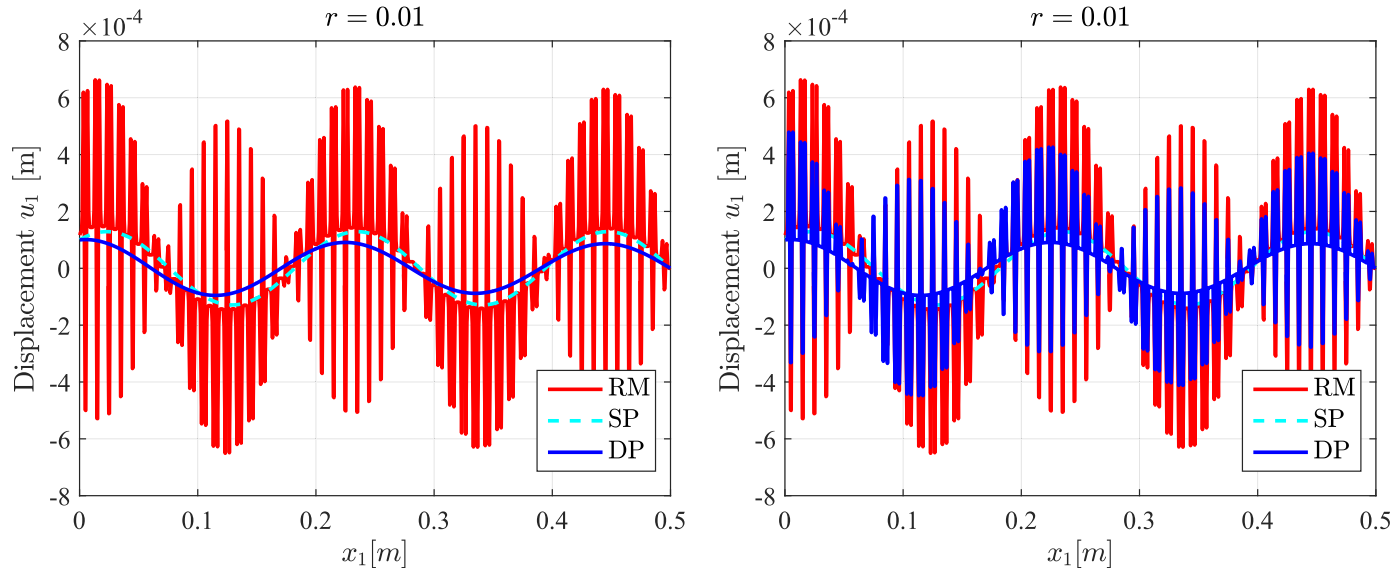

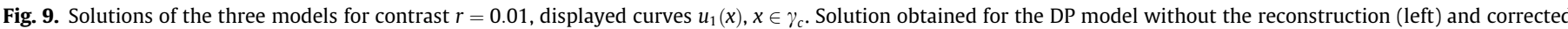
solution $u_{1}^{\mathrm{cr}}$ obtained by the DP model with reconstruction (right).
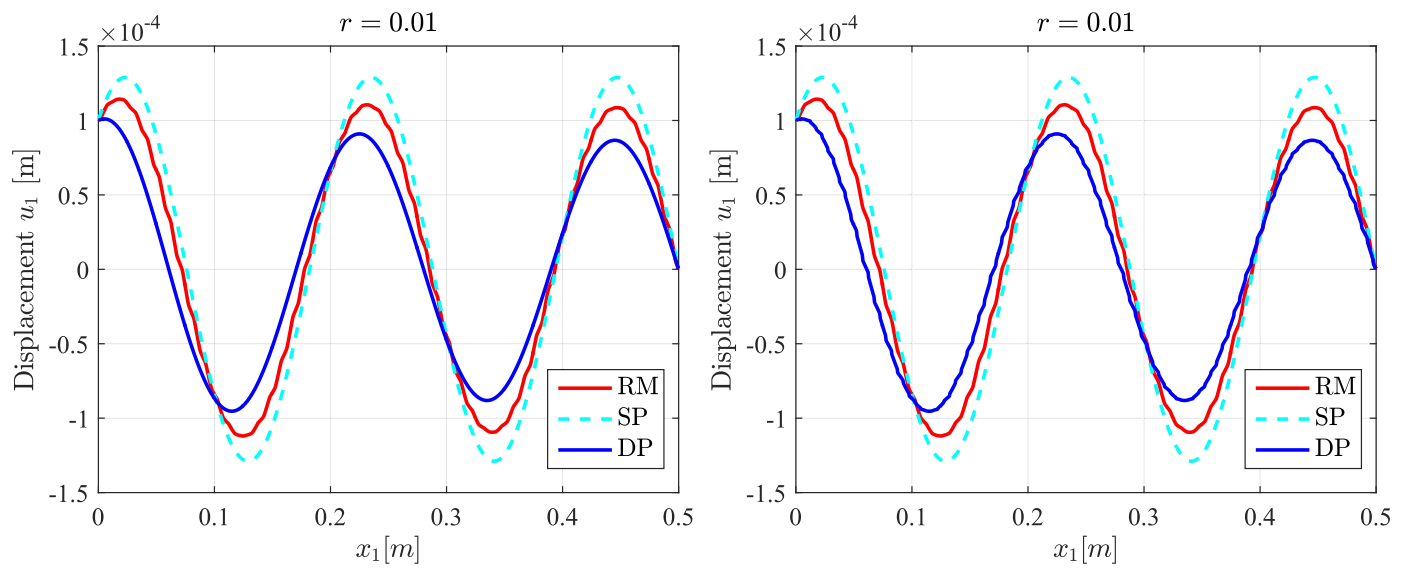

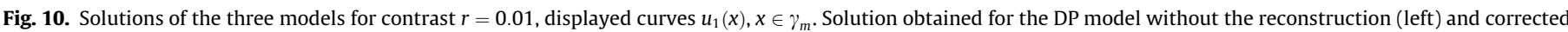
solution $u_{1}^{\text {cr }}$ obtained by the DP model with reconstruction (right).

heterogeneous porous media. As a reference model (RM), we employed the heterogeneous medium described at the "mesoscopic level" using the Biot model and discretized using the finite element method (FEM). The performed numerical tests demonstrate that for large contrast, the DP model with the twoscale reconstruction of the mesoscopic fields provides a very good approximation of the direct simulations, while the SP homogenized model fails. 

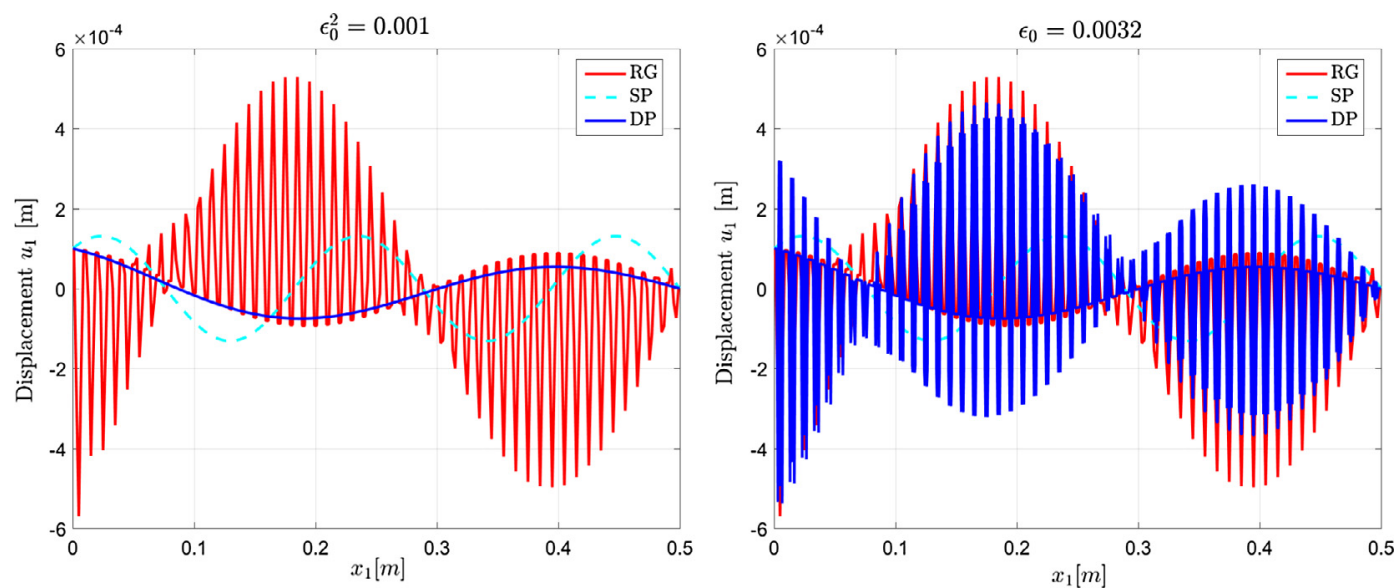

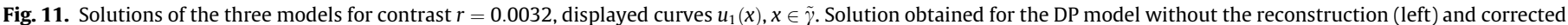
solution $u_{1}^{\text {cr }}$ obtained by the DP model with reconstruction (right).
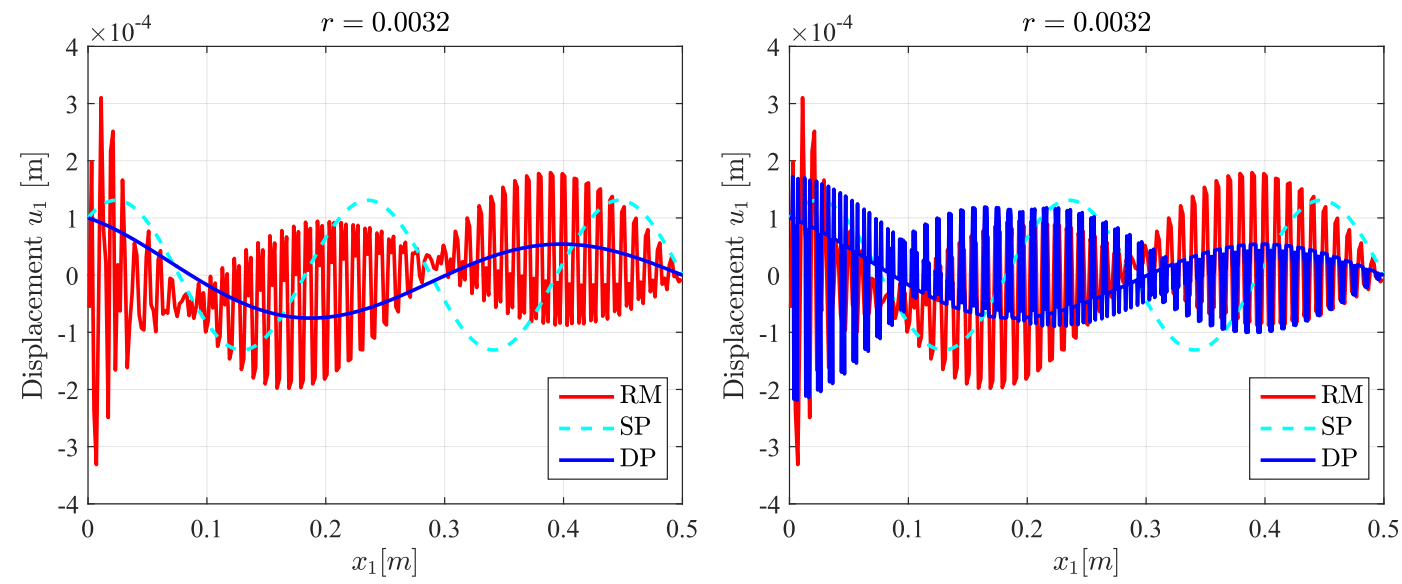

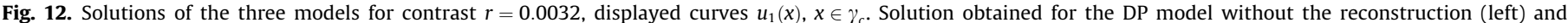
corrected solution $u_{1}^{\mathrm{cr}}$ obtained by the DP model with reconstruction (right).
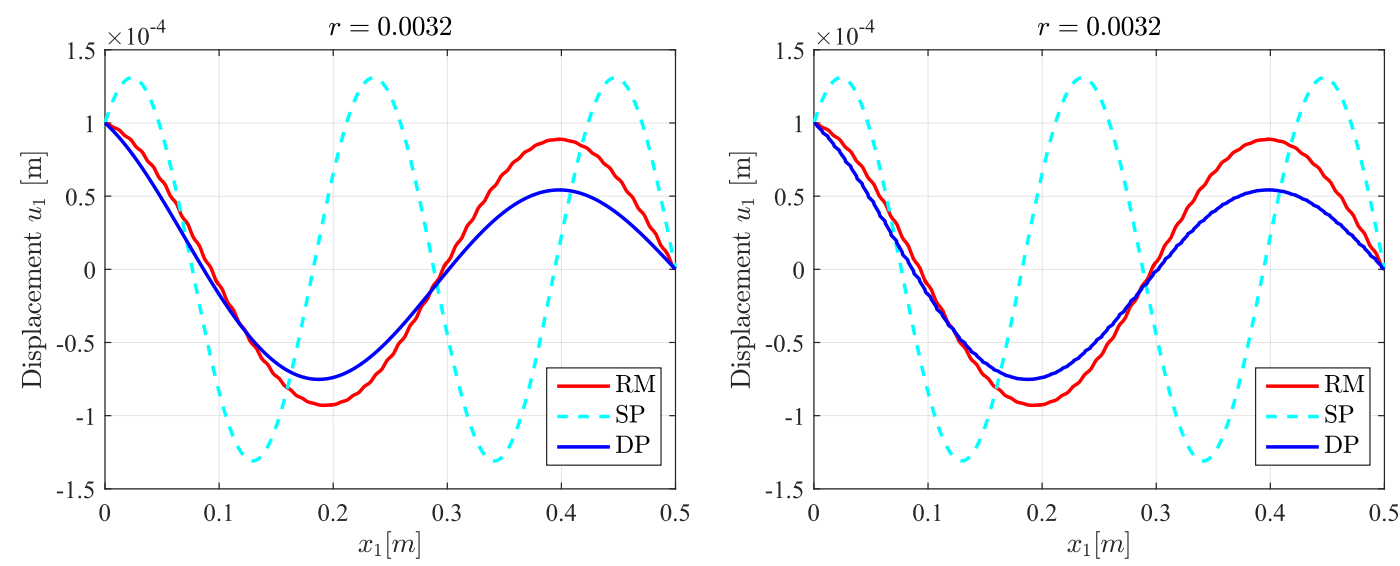

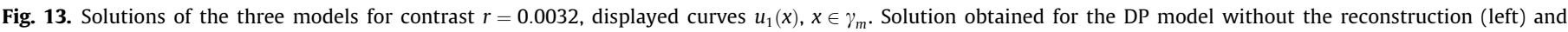
corrected solution $u_{1}^{\text {cr }}$ obtained by the DP model with reconstruction (right).

Especially when solving the wave propagation problem, both the DP and SP models are useful due to much lower computational requirements than those of the FEM models of a heterogeneous, i.e. a non-homogenized medium, which typically lead to a killing complexity even for relatively simple geometries. Moreover, whereas full 3D direct simulations are out reach, the homogenized models provide suitable approximations. In this respect, the choice of one of the models depends on the contrast property of the heterogeneous medium. As pointed out above, the reconstruction of macroscopic responses of the DP model are needed, as amplitudes of the "fluctuations" may be larger than amplitudes of the macroscopic solution. We recall that all the presented study is related to 
the low frequency regime, i.e. the macroscopic wave lengths are significantly larger than the characteristic heterogeneity size. The above mentioned fluctuations are generated by the soft parts of the so-called channels and should not be confused with oscillations observed at the heterogeneity level but induced by high frequency modes, cf. [11]. Our test was limited by the computational power required to solve the direct problem. Similar test should be done for more complex heterogeneous structures and other types of incident waves. Computational accuracy of obtained RM solutions should be verified, however, since the solutions of the RM model may be polluted by numerical errors. Our future research is targeted to explore dispersion properties of the double porosity media described using the presented DP model.

\section{Acknowledgment}

The research of E. Rohan is supported by the project LO 1506 of the Czech Ministry of Education, Youth and Sports, and recently by the grant project GACR 17-01618S of the Czech Science Foundation. A part of the work was done during his stay at the "Université Paris-Est Créteil" (UPEC, France) being financially supported by UPEC and CNRS (France). V.-H. Nguyen and S. Naili have been supported by the Universite Paris-Est and CNRS through the PEPS program (15R03051AMETCARMAT).

\section{Appendix A. The single porosity model}

The homogenized model of the single porosity FSPM was developed in [15]. Here we present the formulations of the local problems solved in the representative cell $Y$. It should be noted, that no decomposition of $Y$ applies in this case, since there is no large contrast in material parameter distribution at the mesoscale.

\section{A.1. Local problems for the characteristic responses}

In analogy with (4.3), (4.4), (3.6), and (3.7), the following analogous formulae hold:

$$
\begin{aligned}
& \begin{aligned}
u^{\varepsilon}(x) & =u^{0}(x)+\varepsilon u^{1}(x, y) \\
& =u^{0}(x)+\varepsilon\left[\omega^{k l}(y) e_{k l}^{x}\left(u^{0}(x)\right)+\omega^{P}(y) p^{0}(x)\right], \\
w^{\varepsilon}(x) & =\lambda \chi^{k}(y)\left(\lambda^{2} u_{k}^{0}(x)+\partial_{k}^{x} p^{0}(x)\right) \\
p^{\varepsilon}(x) & =p^{0}(x)+\varepsilon p^{1}(x, y) \\
& =p^{0}(x)+\lambda \varepsilon \pi^{k}(y)\left(\lambda^{2} u_{k}^{0}(x)+\partial_{k}^{x} p^{0}(x)\right) .
\end{aligned}
\end{aligned}
$$

The characteristic response functions satisfy three local problems:

1. Find $\boldsymbol{\omega}^{k l} \in \mathbf{H}_{\#}^{1}(Y)$, such that for $k=1,2,3$,

$$
a_{Y}\left(\omega^{k l}, v\right)=-a_{Y}\left(\Pi^{k l}, v\right) \quad \forall v \in \mathbf{H}_{\#}^{1}(Y) .
$$

The symmetry of $\omega^{i j}=\omega^{j i}$ is obvious, recalling $\Pi_{k}^{i j}=y_{j} \delta_{i k}$.

2. Find $\boldsymbol{\omega}^{P} \in \mathbf{H}_{\#}^{1}(Y)$, such that

$$
a_{Y}\left(\omega^{P}, v\right)=b_{Y}(1, v) \quad \forall v \in \mathbf{H}_{\#}^{1}(Y) .
$$

3. Given a real frequency $\omega>0$, find $(\boldsymbol{\chi}, \pi) \in \mathbf{L}^{2}(Y) \times H_{\#}^{1}(Y)$ such that for $k=1,2,3$,

$$
\begin{aligned}
\mathrm{i} \omega & \left\langle\boldsymbol{\rho} \boldsymbol{\chi}^{k}, \boldsymbol{\psi}\right\rangle_{Y}+c_{Y}(\boldsymbol{\chi}, \boldsymbol{\psi})+\left\langle\nabla_{y} \pi^{k}, \boldsymbol{\psi}\right\rangle_{Y} \\
= & \frac{\mathrm{i}}{\omega}\left\langle 1_{k}, \boldsymbol{\psi}\right\rangle_{Y},\left\langle\boldsymbol{\chi}^{k}, \nabla_{y} q\right\rangle_{Y}
\end{aligned}
$$

for all $(\psi, q) \in \mathbf{L}^{2}(Y) \times H_{\#}^{1}(Y)$. Using the dynamic permeability $H(\omega)=\left(\mathrm{i} \omega \boldsymbol{\rho}+K^{-1}\right)^{-1}$ and the substitution of
$\chi^{k}=-H(\omega) \nabla_{y}\left(\pi^{k}-\frac{i}{\omega} y_{k}\right)$ into the second equality, the reduced problem for $\pi^{k} \in H_{\#}^{1}(Y)$ can be solved:

$$
\left\langle H(\omega) \nabla_{y}\left(\pi^{k}-\frac{\mathrm{i}}{\omega} y_{k}\right), \nabla_{y} q\right\rangle_{Y_{c}}=0 \quad \forall q \in H_{\#}^{1}(Y) .
$$

Using the characteristic responses we can compute the homogenized permeability and the poroelastic coefficients:

$$
\begin{aligned}
& \mathcal{K}_{k l}^{S}(\omega)=-\mathrm{i} \omega \int_{Y} \chi_{l}^{k} \\
&=-\omega^{2}\left\langle H(\omega) \nabla_{y}\left(\pi^{k}-\frac{\mathrm{i}}{\omega} y_{k}\right), \nabla_{y}\left(\pi^{l}-\frac{\mathrm{i}}{\omega} y_{l}\right)\right\rangle_{Y} \\
&=\mathcal{K}_{l k}(\omega), \\
& \mathcal{M}_{k l}^{S}(\omega)=\int_{Y} \bar{\rho} \delta_{k l}-\mathrm{i} \omega\left(\rho^{f}\right)^{2} \mathcal{K}_{k l}(\omega), \\
& \mathcal{D}_{i j k l}^{S}=a_{Y}\left(\omega^{k l}+\Pi^{k l}, \omega^{i j}+\Pi^{i j}\right), \\
& \mathcal{B}_{i j}^{S}=b_{Y}\left(1, \Pi^{i j}\right)-\mathrm{i} \omega a_{Y}\left(\omega^{P}, \Pi^{k l}\right), \\
& \mathcal{H}^{S}=\int_{Y} \mu^{-1} .
\end{aligned}
$$

The simple porosity homogenized porous medium is governed by Eqs. (3.17), where the above coefficients are substituted and $\underline{\mathcal{C}}=0$.

\section{References}

[1] Arbogast T, Douglas J, Hornung U. Derivation of the double porosity model of single phase flow via homogenization theory. SIAM J Math Anal 1990;21:823-36.

[2] Auriault J-L, Boutin C. Deformable porous media with double porosity III Acoustics. Transp Porous Media 1994;14:143-62.

[3] Auriault J-L, Borne L, Chambon R. Dynamics of porous saturated media, checking of the generalized law of Darcy. J Acoust Soc Am 1985;77 (5): $1641-50$.

[4] Auriault J-L, Boutin C, Geindreau C. Homogenization of coupled phenomena in heterogenous media. Wiley-ISTE; 2010.

[5] Biot MA. Theory of propagation of elastic waves in a fluid-saturated porous solid. I. Low-frequency range. J Acoust Soc Am 1956;28(2):168-78.

[6] Biot MA. Theory of propagation of elastic waves in a fluid-saturated porous solid. II. Higher-frequency range. J Acoust Soc Am 1956;28(2):179-91.

[7] Biot MA. Mechanics of deformation and acoustic propagation in porous media. J Appl Phys 1962;33(4):1482-98.

[8] Brezis H. Functional analysis. Sobolev spaces and partial differential equations. New York: Springer; 2010.

[9] Burridge R, Keller JB. Poroelasticity equations derived from microstructure. J Acoust Soc Am 1981;70:1140-6.

[10] Clopeau T, Ferrín JL, Gilbert RP, Mikelić A. Homogenizing the acoustic properties of the seabed, part ii. Math Comp Model 2001;33:821-41.

[11] Craster RV, Kaplunov J, Pichugin AV. High-frequency homogenization for periodic media. Proc R Soc. A: Math, Phys Eng Sci 2010;466(2120):2341-62. https://doi.org/10.1098/rspa.2009.0612. ISSN 1364-5021.

[12] Ferrín JL, Mikelić A. Homogenizing the acoustic properties of a porous matrix containing an incompressible inviscid fluid. Math Meth Appl Sci 2003;26:831-59.

[13] Griso G, Rohan E. On the homogenization of a diffusion-deformation problem in strongly heterogeneous media. Ricerche Mat 2007;56:161-88.

[14] Hornung U. Homogenization and porous media. Interdisciplinary applied mathematics, vol. 6. Springer; 1996.

[15] Mielke A, Rohan E. Homogenization of elastic waves in fluid-saturated porous media using the Biot model. Math Models Meth Appl Sci 2013;23:873-916.

[16] Nguyen V-H, Rohan E, Naili S. Multiscale simulation of acoustic waves in homogenized heterogeneous porous media with low and high permeability contrasts. Int J Eng Sci 2016;101:92-109.

[17] Norris AN. On the viscodynamic operator in Biot's equations of poroelasticity. J Wave-Material Interact 1986;1:365-80.

[18] Rohan E. Homogenization of acoustic waves in strongly heterogeneous porous structures. Wave Motion 2013;50:1073-89.

[19] Rohan E, Naili S, Cimrman R, Lemaire T. Multiscale modeling of a fluid saturated medium with double porosity: relevance to the compact bone. J Mech Phys Solids 2012;60:857-81.

[20] Rohan E, Naili S, Nguyen V-H. Wave propagation in a strongly heterogeneous elastic porous medium: homogenization of Biot medium with double porosities. Comptes Rendus Mecanique 2016;344(6):569-81.

[21] Rohan E, Naili S, Nguyen V-H. Modelling of waves in fluid-saturated media with high contrast heterogeneity: homogenization approach; 2017 [submitted for publication]. 